\title{
Familia, empleo femenino y reproducción en España: incidencia de los factores estructurales
}

\author{
Almudena Moreno Mínguez \\ Universidad de Valladolid. Departamento de Sociología y Trabajo Social \\ almudena@soc.uva.es
}

Recibido: 13-07-2010

Aceptado: 06-06-2011

\section{Resumen}

El objetivo general de este artículo consiste en presentar las tendencias comparadas relativas al empleo femenino y la reproducción en Europa en relación con las tipologías familiares. En el marco de este objetivo general, el trabajo se propone, por una parte, estimar los factores que mejor explican el declive del modelo familiar de varón sustentador en los países europeos seleccionados. Por otra parte, y en relación con lo anterior, el artículo analiza en qué medida ciertos factores estructurales explican, desde un enfoque transversal, la reducida fecundidad existente en España, así como la probabilidad de que la mujer esté empleada. Con tal fin, se han utilizado los datos agregados procedentes de diferentes fuentes europeas, así como los datos de la European Labour Survey 2006 y la Encuesta de Familia y Empleo 2006. Los resultados presentados en este trabajo apuntan a que la relación entre educación, empleo femenino y fecundidad en contextos de limitado apoyo institucional a los padres es determinante en España. Se ha comprobado también que el modelo familiar de dos sustentadores económicos se asocia, en España, con un reducido número de hijos y un nivel educativo medio y alto de los miembros de la pareja, mientras que tal asociación es menos significativa en otros países europeos con contextos institucionales más favorables al empleo femenino y la conciliación laboral y familiar.

Palabras clave: cambio familiar; fecundidad; ocupación; género; Europa.

Abstract. Family, women's employment and reproduction in Spain: The impact of structural factors

The objective of this article is to present comparative trends regarding female employment and fecundity in Europe in relation to family typologies. This study estimates the factors that best explain the decline in the male breadwinner family model in several European countries. Furthermore, the article analyses to what extent certain structural factors transversally explain reduced fecundity in Spain and the probability of women being employed. Aggregate data have been used from various European sources such as the 2006 European Labour Force Survey and the Encuesta de Familia y Empleo 2006 (2006 Family and Employment Survey). Our results suggest that the relationship between education, female employment and fecundity in a context of limited institutional support for parents 
is a determining factor in Spain. The family model of two breadwinners in Spain was also found to be associated with a reduced number of children and medium to high educational levels in couples. This correlation was less significant in other European countries with institutional contexts that are more favourable to female employment and the reconciliation of work and family life.

Keywords: family change; fertility; labour; gender; Europe.

\begin{tabular}{|c|c|}
\hline \multicolumn{2}{|c|}{ Sumario } \\
\hline Introducción & Análisis de los factores explicativos \\
\hline $\begin{array}{l}\text { Marco teórico. Explicando el declive } \\
\text { de la fecundidad en un contexto } \\
\text { de creciente participación laboral }\end{array}$ & $\begin{array}{l}\text { del empleo femenino y la reproducción } \\
\text { en España en un contexto de cambio } \\
\text { familiar }\end{array}$ \\
\hline femenina y cambio familiar & Conclusiones \\
\hline Objetivos, hipótesis y metodología & Referencias bibliográficas \\
\hline
\end{tabular}

\section{Introducción}

Son numerosos los análisis empíricos comparados que han analizado la asociación entre fecundidad y empleo femenino en los diferentes regímenes de bienestar, subrayando la relevancia de las políticas familiares. Sin embargo, son menos frecuentes los estudios que han analizado la asociación entre el empleo femenino y la fecundidad en relación con las tipologías familiares en función de factores individuales tales como la educación, la edad o los ingresos.

Este artículo tiene como objetivo analizar la posible interconexión existente a nivel macro entre las tipologías familiares y laborales (un sustentador o dos sustentadores económicos) y el comportamiento reproductivo de las parejas en España, partiendo de la hipótesis de que la progresiva incorporación de la mujer al mercado laboral se encuentra vinculada, en mayor o menor medida, a factores tales como el número y la edad de los hijos, la formación y la edad, dependiendo del contexto institucional de referencia. La literatura científica existente a tal efecto ha demostrado que los efectos de las políticas familiares sobre la fecundidad es diversa, puesto que depende del contexto institucional y de cada hogar, aunque sí que parece ser más evidente la asociación positiva entre empleo femenino y políticas familiares, con el consiguiente efecto sobre la reproducción (Da Rocha y Fuster, 2006; Neyher, 2003).

No es objeto de estudio en este artículo analizar la vinculación entre las políticas familiares, el empleo femenino y la fecundidad, ya que existe una amplia literatura al respecto, ni tampoco analizar las preferencias, las actitudes y los valores ante la maternidad y el empleo, ya que estos estudios requieren otro tipo de análisis. Tampoco es objeto de este trabajo analizar pormenorizadamente la fecundidad a través de los múltiples indicadores existentes para ello y las complejas técnicas utilizadas por los demógrafos, sino que el objeto de este 
análisis ha sido estudiar en qué medida actúan variables tales como la formación de los miembros de la pareja y el número de hijos en el empleo femenino y, por tanto, en las tipologías familiares existentes en cada país en contextos institucionales diferenciales. Es decir, el artículo trata de buscar algún tipo de asociación contextual en la asociación existente entre el empleo femenino, el cambio familiar y la reproducción, sin ánimo de establecer la exhaustividad estadística que se deriva de los sofisticados análisis realizados por los demógrafos. En este artículo, partimos de la evidencia, demostrada en numerosos artículos científicos, de que España pertenece al grupo de países denominados «lowest.low fertility countries» (Kohler et al., 2006), con una reducida fecundidad, en donde la progresiva incorporación de la mujer al mercado laboral no se ha traducido en un incremento de la fecundidad, sino más bien en el mantenimiento de estos reducidos niveles, sobre todo en las mujeres con mayor grado educativo que retardan el nacimiento del primer hijo y reducen la fecundidad total en mayor medida que las mujeres con reducido nivel educativo (Martín García, 2009; Baizán y Martín García, 2007). De hecho, y como hipótesis de partida, podríamos decir que el lento pero progresivo incremento del modelo familiar de dos sustentadores económicos en España se asocia con una reducida fecundidad y niveles educativos medios y altos de los miembros de la pareja, mientras que el modelo familiar de varón sustentador se asocia con mayor número de hijos y niveles bajos de formación entre los miembros de la pareja. Si bien este fenómeno no ha sido muy estudiado desde esta perspectiva familiar, sí que ha sido estudiado desde el prisma de la incidencia que han tenido las políticas familiares en el empleo femenino y la fecundidad, temática que no se abordará en este trabajo porque ya ha sido ampliamente tratada por la literatura. Sin embargo, este trabajo sí que parte de estos hallazgos empíricos previos que han constatado como el contexto de reducidas políticas familiares en países como España e Italia ha dificultado la compatibilización familiar y laboral y, por tanto, el empleo femenino, con lo cual este hecho ha tenido repercusiones probadamente negativas en la fecundidad (Del Boca et al., 2004; Moreno Mínguez, 2007, Castles, 2003; Thévenon, 2009).

Para llevar a cabo tal ejercicio empírico, en primer lugar, se realiza un análisis descriptivo general comparado, con el fin de identificar tendencias diferenciadas entre los países objeto de estudio en cuanto al empleo femenino y a las tipologías familiares y laborales según número de hijos y edad de los mismos. A este respecto, partimos del hecho constatado por numerosos análisis empíricos de que, en los países del sur de Europa, y concretamente en España, contrariamente al resto de los países de la Europa de los quince, la creciente incorporación de la mujer al mercado laboral a partir de los años ochenta no ha ido pareja a un incremento en la fecundidad, sino que esta se ha mantenido a niveles reducidos (Da Rocha y Fuster, 2006; Del Boca et al., 2004; Moreno Mínguez, 2007).

Puesto que se han realizado numerosos trabajos empíricos para testar la importancia de las políticas familiares en la explicación de este fenómeno, el propósito de ese artículo en un segundo apartado no es este, sino analizar, por una 
parte, los factores que mejor predicen la fecundidad y se asocian con los modelos familiares de varón sustentador y de dos sustentadores económicos en los países europeos para los que había datos disponibles procedentes de la European Labour Survey para el año 2006. Por otro lado, se analiza la capacidad predictiva de ciertas variables de índole individual, tales como la edad, la formación, la renta, el modelo de familia y el grado de dificultad para compatibilizar familia y empleo, entre otras variables, en la reproducción. También se analiza qué factores, entre los disponibles en la fuente utilizada, predicen mejor el empleo femenino en España. Para ello, se ha realizado un análisis transversal mediante el desarrollo de modelos de regresión a partir de los datos de la Encuesta de Familia y Empleo 2006, realizada en el marco de un proyecto de investigación.

Por lo tanto, el artículo se divide en tres partes diferenciadas: en una primera parte, se presenta el análisis del marco teórico en el que se contextualiza el objeto de estudio y las hipótesis. A continuación, se presenta un breve análisis descriptivo comparado sobre los comportamientos reproductivos y las tipologías familiares, así como de las tendencias de empleo femenino según número y edad de hijos a cargo. En el último apartado, se analizan los resultados de los modelos de regresión desarrollados por los países europeos para los que había datos disponibles y para España.

\section{Marco teórico. Explicando el declive de la fecundidad en un contexto de creciente participación laboral femenina y cambio familiar}

Los modelos teóricos desarrollados para analizar el cambio familiar han enfatizado el cambio experimentado en la posición económica y social de las mujeres como catalizadoras de las estrategias familiares, laborales y reproductivas de las parejas en los diferentes países europeos. En la mayoría de los países europeos occidentales, la evolución de la fecundidad se ha producido de forma paralela a los cambios experimentados en la posición de la mujer en el mercado laboral y en la familia, así como en las políticas sociales y familiares. Esto explicaría en parte la persistencia de la correlación negativa entre fecundidad y empleo femenino, constatada por los diferentes estudios empíricos realizados hasta la década de los ochenta, momento en el que esta asociación se torna positiva para la mayoría de los países europeos (OCDE, 2007; Castles, 2003; Moreno Mínguez, 2007). Sin embargo, en el caso de los países del sur de Europa, fundamentalmente España e Italia, la reducción experimentada por la fecundidad desde 1975 se ha producido en contextos familiares de reducida participación laboral femenina, en los que ha primado el modelo familiar de varón como principal sustentador. Los debates y los análisis surgidos en torno al cambio en las tipologías familiares en relación con la evolución del empleo femenino han suscitado una gran controversia teórica, como se puede ver en los múltiples trabajos realizados a este respecto (Hakim, 2000; Crompton, 2006; Duncan, 2003), aunque son menos los estudios que se han realizado sobre el cambio en las tipologías familiares en relación con las pautas reproductivas y demográficas (Ranci, 2010). En este trabajo, entendemos por tipologías familiares 
aquellas formas de hogar que se definen por la dedicación de los miembros de la pareja al trabajo remunerado y al cuidado familiar (care). Partiendo de esta definición, la literatura científica ha diferenciado entre el male bread winner model ('modelo de varón sustentador') o el single earnes familias (Ranci, 2010) y el denominado adult-worker model family ('modelo de dos sustentadores económicos') (Lewis, 2002). Generalmente, se ha asociado la evolución de estas tipologías familiares con los mecanismos institucionales y familiares que se han desarrollado para solventar los denominados «problemas de conciliación laboral y familiar». De esta forma, los investigadores se han referido a las tipologías de las políticas familiares (Boje y Leira, 2000; Wall, 2007), a los regímenes de cuidado (care regimes) (Bettio y Plantenga, 2004), al régimen de Estado de bienestar (Sainsbury, 1999) y al régimen de género (gender regime) (Pascall y Lewis, 2001), con el fin de tratar de explicar y comparar los diferentes modelos familiares presentes en cada contexto institucional.

En cierta medida, la permanencia de la asociación negativa entre fecundidad y empleo femenino en un contexto familiar de lento declive del modelo de varón sustentador en países como España cuestiona en parte los postulados de la teoría de las preferencias (Hakim, 2000) ${ }^{1}$, ya que apunta a posibles factores estructurales tales como: los determinantes económicos (el coste de oportunidad laboral), institucionales (políticas familiares), formativos (nivel educativo) y culturales (roles de género). En concordancia con esta línea de interpretación, numerosos investigadores han subrayado que las decisiones individuales adoptadas en torno a la reproducción, el empleo y la formación de la familia están en cierta manera influenciadas por los contextos sociales y culturales en los que viven los individuos (Delgado et al., 2009; De Laat y Sevilla, 2006; Moreno Mínguez, 2007; Fernández et al., 2004; Fernández y Fogli, 2009; Valiente, 2010).

Por otra parte, contamos con numerosas teorías que han tratado de explicar la evolución de la fecundidad en Europa en relación con el cambio familiar desde los años sesenta desde una perspectiva sociológica. Los teóricos de la segunda transición demográfica entienden que el declive en la fecundidad es el resultado de la pluralización de las formas familiares convergentes en Europa (Van de Kaa, 1987). Sin embargo, la reducida fecundidad que se mantiene en los países del sur de Europa desde mediados de los años setenta contradice esta tesis demográfica de la convergencia, ya que en los países del sur de Europa es precisamente donde menor es la pluralización de los estilos y las formas de vida familiares y de las formas familiares de dos sustentadores (Billari y Wilson, 2001) y mayor la dependencia de formas tradicionales de organización familiar (Blossfeld y Drobnic, 2001; Naldini, 2003). Para explicar la paradójica coexis-

1. Hakim (2000) ha clasificado las preferencias de las mujeres del siglo XXI en relación con los estilos de vida frente al trabajo y la familia:

- Centrada en el hogar: la vida de familia y los niños son sus principales prioridades.

- Adaptativa: este grupo es mayoritario e incluye a aquellas mujeres que quieren compatibilizar trabajo y familia, sin dar prioridad al trabajo profesional.

- Centrada en el trabajo: se trata mayoritariamente de mujeres sin hijos cuya prioridad fundamental es el trabajo profesional. 
tencia de pautas demográficas de fecundidad reducida con formas familiares tradicionales en Europa del sur (Zuanna y Micheli, 2004), los investigadores han resaltado la debilidad del Estado de bienestar y la importancia de la solidaridad familiar como red de protección social (Esping-Andersen y Sarasa, 2002; Moreno Mínguez, 2007); la elevada temporalidad y desempleo de los jóvenes (Ahn y Mira, 2001; González y Jurado, 2006), así como el inflexible e inaccesible mercado de la vivienda (Kohler et al., 2006).

También se ha señalado como posible causa interviniente en este hecho la desigualdad existente en las relaciones de género en el ámbito privado familiar, que no se corresponde con las expectativas de igualdad generadas en el ámbito público (McDonald, 2000). Se ha escrito numerosa literatura sobre el cambio en los roles de género en relación con el advenimiento de las nuevas formas de familia y la división del trabajo familiar. A este respecto, destacan las teorías relativas a la socialización de los roles de género, según las cuales las mujeres reproducen ciertas preferencias sobre los estilos de vida y roles de género más orientados al trabajo o a la familia en función del proceso de socialización (Hakim, 2000). Esta teoría ha sido cuestionada por investigadores que defienden la incidencia de los factores estructurales en la conformación de la preferencias, tales como la clase social, el estatus ocupacional, etc. (Crompton y Lyonette, 2010; Bittman et al., 2003; Breen y Coke, 2005).

En el marco de esta perspectiva interpretativa, destaca la teoría del doing gen$d e r^{2}$, que destaca el significado simbólico de los roles de género para explicar la desigual división del trabajo doméstico. Rissman (2004), en un intento por unir la perspectiva culturalista e institucionalista, consideró que los roles de género responden a una forma de estructura social o estratificación social inserta en el entramado cultural, lo que explica las diferencias y las oportunidades en función de la adscripción de sexo. Si bien estas interpretaciones no han conseguido dar una explicación convincente sobre la relación existente entre los roles de género, el cambio familiar y el empleo femenino en los diferentes contextos institucionales, sí que han servido de plataforma para ensayar interpretaciones acerca de cómo los roles de género median o interfieren en las actitudes y los valores hacia el empleo y la fecundidad. Cooke (2009) ha demostrado como contextos institucionales en los que se ha favorecido cierta igualdad en los roles de género, tanto en el ámbito familiar como público, la fecundidad y el empleo femenino han alcanzado mayores índices que en otros contextos menos generosos hacia la igualdad de género y la corresponsabilidad familiar.

Aunque el análisis empírico realizado en este trabajo no se ocupa explícitamente de analizar los roles de género en relación con las tendencias de cambio familiar, debido fundamentalmente a que los datos utilizados no nos permiten

2. Según esta perspectiva, la construcción simbólica del género asociada con las pautas de división del trabajo es básicamente una práctica social que se crea y se reconstituye a través de la interacción social de hombres y mujeres, así como a través de las acciones de las instituciones como el mercado laboral, el Estado de bienestar y la propia incidencia de la cultura familiar (Coltrane, 2000; West y Zimmerman, 1987). 
operativizar adecuadamente este concepto, sí que se utiliza este factor como elemento transversal de los cambios que están aconteciendo en las tipologías familiares, en el empleo femenino y en la reproducción en los diferentes contextos institucionales. De hecho, los estudios realizados a este respecto apuntan a que, en los países del sur de Europa, se está produciendo un gradual y contradictorio cambio en los roles de género que está teniendo una clara incidencia en las pautas reproductivas, familiares y laborales, sobre todo de la población femenina (Lück y Hofäcker, 2008: 301; Castro et al., 2008; Parella, 2008).

En los últimos años, y en relación con la teoría de los roles de género, se ha incorporado a estas explicaciones el indicador referido al rol desempeñado por el varón en la realización de las tareas domésticas, sobre todo como factor predictivo para tener un segundo o un tercer hijo (Cooke, 2001, 2009; Brodmann et al., 2007; González y Jurado, 2009). En el caso de los países nórdicos como Suecia, se ha constatado el hecho de que una mayor igualdad en la realización de las tareas domésticas y en la compartición de los permisos parentales entre los miembros de la pareja tiene ciertos efectos positivos sobre la fecundidad (Bernhardt y Goldscheider, 2008; Oláh, 2003; Escobedo, 2008). Similares resultados, utilizando diferentes modelos, datos y métodos de análisis, se han obtenido para Noruega (Duvander, 2008), España (De Laat y Sevilla Sanz, 2006), así como para Dinamarca (Feyrer et al., 2008). Sin embargo, y a pesar de esta sólida literatura empírica, los resultados son contradictorios y, por lo tanto, no concluyentes. Así, por ejemplo, según Puur et al. (2008), las actitudes igualitarias de los hombres están asociadas con una elevada fecundidad, mientras que según Westoff y Higgins (2009), por el contrario, las actitudes igualitarias de los hombres están asociadas con una reducida fecundidad. En definitiva, estas contradicciones subrayan la necesidad de seguir profundizando en este tipo de análisis desde una perspectiva comparada, teniendo en cuenta la especificidad cultural e histórica de cada país (Goldscheider et al., 2010).

En cualquier caso, las teorías que más fuerza han cobrado a lo largo de estos últimos años para explicar el cambio de signo en la asociación entre la fecundidad y el empleo femenino en Europa a partir de los años ochenta son las que se refieren a la incidencia de las políticas familiares. Estos estudios han analizado, desde perspectivas diferentes, la incidencia de las políticas públicas en la fecundidad y en el empleo femenino en los distintos países europeos, sin embargo, ninguno de ellos ha resultado ser concluyente. La idea de que las políticas públicas pueden activar la fecundidad ha sido replicada con el argumento, defendido por numerosos investigadores, de que este tipo de políticas pronatalistas, consideradas aceptables por muchos gobiernos europeos, han resultado ser muy caras e inefectivas. La futilidad de las políticas públicas como herramienta para activar la fecundidad en Europa ha sido especialmente destacada por el investigador Demeny (2003), quien considera que los mecanismos homeostáticos de carácter endógeno de la reproducción hacen innecesarios los esfuerzos pronatalistas de los gobiernos. Por otro lado, la idea de que las políticas familiares tienen una incidencia muy limitada en la fecundidad ha sido analizada recientemente por Gauthier (2007: 339), quien ha manifestado 
cierta dificultad para comprender la razón de por qué los «cheques bebé»son tan populares entre algunos gobiernos europeos. Según Gauthier, los efectos de las políticas públicas sobre la reproducción son muy reducidos y en ningún caso afectan a la secuencia de los nacimientos ni al tamaño de la familia. Si bien los estudios empíricos realizados no han encontrado asociaciones significativas entre la evolución de la fecundidad y las transferencias directas destinadas a las familias (por ejemplo: los cheques por nacimiento) en los países de la OCDE y en los países europeos, sin embargo, sí se han encontrado asociaciones de signo positivo entre la fecundidad y los gastos destinados a servicios familiares de atención a la infancia (Castles, 2003; Moreno Mínguez, 2007).

Los economistas y los sociólogos han sido también muy cautos en señalar la posible incidencia de las políticas económicas sobre la fecundidad y han preferido centrarse en el análisis de cómo las políticas públicas pueden contribuir a favorecer el empoderamiento de las mujeres mediante el empleo y, por tanto, incidir indirectamente sobre la activación de la fecundidad (Esping-Andersen y Sarasa, 2002; Lewis, 1992; Gornick et al., 1997; Sainsbury 1999; Anttonen y Sipilä, 1996; Castles, 2003; Neyer, 2003). A este respecto, contamos con sólidos análisis empíricos que avalan la teoría de que una política que apoya la igualdad de género en el ámbito público contribuye a la igualdad de género en el ámbito familiar privado, lo cual contrarresta en parte los efectos negativos de la fecundidad asociados con el empleo femenino (Cooke, 2009; Oláh, 2003; Torr y Short, 2004). No es objeto de este artículo seguir indagando en la posible incidencia de las políticas públicas en el empleo femenino ni en los efectos que estas tienen indirectamente sobre la fecundidad, sino únicamente tomarlo como punto de partida para analizar, desde una perspectiva macro, la posible asociación entre el empleo femenino, la fecundidad y las tipologías familiares en el contexto de posiciones individuales diferenciadas por la edad, la educación o la renta.

En este complejo mosaico de asociaciones, la educación desempeña un papel fundamental, ya que la formación, además de proveer credenciales individuales para ajustar la incorporación al mercado laboral, favorece también la formación integral del individuo en lo que se refiere a expectativas, motivaciones y aspiraciones. Por lo tanto, además de la constatada incidencia de los factores institucionales, ampliamente discutida en la literatura científica (Ronsen y Skrede, 2010), los factores estructurales como la educación parecen tener también una incidencia clave, tanto en la fecundidad, como en la participación laboral de la mujer. Según los estudios empíricos realizados a tal efecto en los países del entorno mediterráneo como Italia, la participación laboral de la mujer depende más de la educación que del número de hijos (Solera, 2009). En los casos de España e Italia, donde las políticas familiares y el mercado de trabajo no han sido especialmente favorables a la inserción laboral de las madres, la asociación entre formación de la mujer y fecundidad es negativa (Martín García, 2010), mientras que en los países del norte de Europa, con políticas más favorables a la inserción laboral de la mujer con cargas familiares, se ha reducido la asociación negativa entre formación femenina y número de hijos. De hecho, investigadores como Andersson et al. (2009) se refieren a un régimen común de fecundidad 
para Dinamarca, Noruega, Finlandia y Suecia, caracterizado por una recuperación de la fecundidad en las mujeres con mayores niveles educativos. Según estos investigadores, esto se debe fundamentalmente al impacto que han tenido las políticas sociales en favorecer la igualdad de género y reducir las diferencias existentes por niveles educativos en el comportamiento reproductivo ${ }^{3}$. La incidencia del nivel educativo en la fecundidad en España ha sido un tema ampliamente estudiado por Martín García (2009) y Baizán y Martín García (2007), quienes han minimizado la incidencia negativa del nivel educativo en la fecundidad en España, debido, según los resultados de sus cálculos, a que en este fenómeno interviene una compleja red de factores, en los que la formación es un elemento más de la interpretación.

Las investigaciones realizadas para España han evidenciado que, en un contexto institucional escasamente favorable para la compatibilización laboral y familiar, el nivel educativo de la mujer interviene de forma indirecta retrasando el nacimiento del primer hijo o espaciando en el tiempo el nacimiento de los hijos como instrumento de conciliación laboral y familiar (Cordero, 2009), pero no necesariamente reduciendo el número total de hijos. De hecho, numerosos investigadores han constatado que el incremento de años de formación contribuye a posponer el nacimiento del primer hijo, pero no necesariamente afecta de manera negativa a la fecundidad completa de una mujer, ya que la educación incrementa las posibilidades de poder «comprar» en el mercado los servicios relativos al cuidado de los dependientes. Bloemen y Kalwij (2001) lo han estudiado para Holanda; Chiuri (2000) y Bratti (2003), para Italia, y Delgado et al. (2009) para España. Si bien son numerosas las investigaciones que han analizado a nivel micro la interrelación de los factores familiares, socioeconómicos y culturales que intervienen en la decisión de tener hijos y en el retraso de la maternidad en España (Delgado et al., 2008), menos habituales son los estudios que tratan de contextualizar a nivel macro la compleja red de factores que intervienen en la maternidad.

Si bien la pretensión de este artículo no es analizar la posible asociación existente entre lo valores culturales familistas tradicionales ${ }^{4}$, el empleo femenino

3. A este respecto, no hay que olvidar los efectos de segregación ocupacional por sexo que han tenido las políticas públicas en los países del norte de Europa.

4. Los valores familistas y su incidencia en el empleo femenino y la fecundidad han sido ampliamente analizados en la literatura científica (Leitner, 2003; Naldini, 2003; Moreno Mínguez, 2007; Flaquer, 2005; Zuanna, 2001). De acuerdo con las interpretaciones antropológicas de Zuanna y Micheli (2004: 15), en el denominado «modelo familista» (family solidarity approach), característico de los países del sur de Europa, la solidez de los vínculos familiares ha tenido un rol central en la organización del proceso social y económico de la reproducción, puesto que la orientación comunitaria y el sentimiento cívico están subordinados al «bien de la familia». Según Zuanna, la continuidad de este sistema normativo explica la reducida fecundidad en estos países, ya que, en un contexto de creciente inestabilidad económica, la solidaridad familiar es la mejor opción para procurar el bienestar futuro de los hijos, y este objetivo sólo es plausible si se reduce el número de ellos. De acuerdo con Zuanna y Micheli (2004: 18): «The very low fertility in southern Europe at the end of twentieth century is thought to be the product of couples' desire to reconcile the co-existence of economic rationality with familist rationality». 
y la maternidad, sí al menos se trata de plantear la posible asociación existente entre el lento pero progresivo cambio hacia un modelo familiar de dos sustentadores económicos y la reducida fecundidad en España, en un contexto de limitadas políticas familiares de conciliación (Flaquer, 2005; Flaquer et al., 2009; Meil, 2006; Moreno Mínguez, 2007) y donde los factores individuales como la educación o la renta pueden incidir de alguna forma en los efectos que tiene la cultura familista sobre la fecundidad. Esta perspectiva, un tanto crítica con la teoría individualista de las preferencias de Hakim, ha abierto una nueva línea de investigación en torno a la incidencia de la cultura familiar en los comportamientos reproductivos y laborales, sobre la que habrá que seguir trabajando en el futuro, puesto que este no es el objetivo de este trabajo.

\section{Objetivos, hipótesis y metodología}

\section{Objetivos e hipótesis}

Este artículo pretende, desde una perspectiva macro y con datos de carácter transversal procedentes de fuentes estadísticas diferentes, indagar desde una perspectiva comparada sobre una posible vía de asociación entre el empleo femenino y la reproducción en un contexto de creciente ascenso de las familias con dos sustentadores económicos y, por tanto, declive de modelo de varón sustentador, teniendo en cuenta indicadores tales como la formación de los miembros de la pareja.

En concreto, los objetivos de los análisis presentados en este trabajo se centran principalmente en explicar, por una parte, los factores que mejor explican la probabilidad de que haya familias con un varón sustentador y familias con dos sustentadores económicos en los diferentes países europeos sobre los que hay datos disponibles. Otro objetivo consiste en analizar los factores que mejor predicen la probabilidad de tener hijos en España observando aspectos tales como la edad, el estado civil, el nivel de estudios, el modelo familiar y la situación laboral de la mujer y, por otra parte, analizar los factores que explican la probabilidad de que la mujer trabaje o no trabaje en un contexto de erosión del modelo familiar de varón sustentador.

En base al marco teórico planteado en este artículo y a los datos comparados disponibles en varias fuentes europeas, tales como la European Labour Survey 2006 y la Encuesta de Familia y Empleo para España 2006, este artículo pretende avanzar en la formulación y la comprobación de las siguientes hipótesis derivadas de los diferentes enfoques teóricos presentados:

- El impacto del número de hijos en los modelos familiares y en el empleo femenino es especialmente relevante en España, donde la presencia de hijos se asocia con el modelo de varón sustentador en mayor medida que en otros países europeos, debido a las dificultades de conciliación laboral y familiar vinculadas con el marco institucional. Por otra parte, en España, la probabilidad de que la mujer trabaje estará especialmente relacionada con su nivel educativo y con el número de hijos. 
- El impacto de la educación en la fecundidad y el empleo femenino es más significativo en aquellos países donde las mujeres tienen mayores dificultades para compatibilizar vida laboral y familiar, debido al contexto institucional de limitadas ayudas públicas. En el caso español, la probabilidad de tener un hijo o más está especialmente asociada con el nivel educativo de la mujer, es decir: a mayor formación, menor número de hijos.

- El modelo familiar de dos sustentadores económicos se asocia en España a un reducido número de hijos y a un nivel educativo medio y alto de los miembros de la pareja, mientras que en otros países europeos, con otros contextos económicos, estos factores no son tan significativos.

\section{Metodología}

La estrategia metodológica desarrollada en este trabajo para conseguir estos objetivos se ha basado principalmente en presentar datos comparados a partir de las informaciones obtenidas de diferentes fuentes europeas. Estos análisis descriptivos se han completado con la aplicación de modelos de regresión comparados a partir de los datos procedentes de la European Labour Survey. Para el caso español, se han realizado también diferentes modelos de regresión logística a partir de los datos de la Encuesta de Familia y Empleo 2006, para predecir factores tales como la educación, la edad o el tipo de familia, que son los que mejor explican la asociación negativa entre fecundidad y empleo femenino existente en España.

Una vez realizado el análisis descriptivo, el artículo desarrolla, a continuación, diferentes modelos de regresión. En un primer análisis, se ha definido un modelo de regresión logística para analizar los factores de tipo individual que mejor explican la probabilidad de que, en los países europeos sobre los que hay datos disponibles, se dé el modelo de varón sustentador y de dos sustentadores económicos. Los datos utilizados para llevar a cabo este análisis se basan en la European Labour Survey hecha en el año 2006. Esta encuesta se ha realizado a una muestra representativa de personas con edades comprendidas entre los 15 y los 64 años (16-64 para España, Reino Unido y Noruega), y se lleva a cabo anualmente para los 27 países de la Unión Europea, con una muestra total de 116.817 casos. En este análisis, se han construido dos variables dependientes a partir de la variable definida en la encuesta (estatus laboral de los miembros de la pareja), que se refieren, por un lado, a los hogares familiares en los que hay un solo sustentador varón y otra variable dependiente en la que el hombre y la mujer trabajan. El grupo de entrevistados seleccionados se refiere a los hogares familiares compuestos por hombres y mujeres entre 20 y 59 años según situación laboral de ambos con y sin hijos. Las variables independientes seleccionadas en el análisis son las referidas al número y a la edad de los hijos, la edad y la formación del hombre y de la mujer, las razones relativas al cuidado de los hijos para que no trabaje la mujer y la ocupación del varón. Para todas las variables independientes, todas ellas categóricas, se ha seleccionado la primera categoría como referencia 
para el análisis estadístico. Sin embargo, para algunos países, se ha tomado la última categoría de las variables sobre la edad y la ocupación del varón, puesto que, cuando se utilizaba la primera categoría como la de referencia, se producían incoherencias, ya que la variable era significativa, pero no lo eran las categorías de análisis en relación con la de referencia. Los países incluidos en el análisis son Austria, Bélgica, Alemania, España, Francia, Grecia, Italia, Países Bajos y Reino Unido. El resto de los países europeos de los quince fueron descartados, porque no contaban con casos suficientes para proceder con el análisis estadístico.

En los análisis de regresión referidos al caso español, se han utilizado los datos procedentes de la Encuesta de Familia y Empleo 2006. Se trata de una encuesta realizada en el marco de un proyecto de investigación en el año 2006 que es representativa de la población española de carácter transversal y que incluye a 800 personas entrevistadas de edades comprendidas entre los 18 y los 55 años $^{5}$. Los encuestados se obtuvieron sobre una muestra de 3.000 internautas, seleccionados a través de cuotas y pertenecientes al panel de la empresa especializada que distribuyó la encuesta. Las cuotas utilizadas para elaborar el presente muestreo se refieren a la edad, el hábitat, las comunidades autónomas, el sexo y el número de hijos. La encuesta recoge datos individuales y referidos al hogar. La recogida de datos se efectuó a partir de un cuestionario que se distribuyó en línea con 45 ítems, proceso que fue realizado por una empresa especializada en este tipo de encuestas a partir del cuestionario facilitado por el equipo de investigación.

Este análisis se ha planteado a partir de la selección de dos variables dependientes para ser explicadas por separado y así evitar los posibles efectos endógenos. Por una parte, se ha definido la variable referida al número de hijos del total de la población encuestada y, por otra, la variable referida a la situación laboral de la mujer. Se ha optado por un análisis de tipo transversal mediante la aplicación del método estadístico de regresión logística como instrumento de análisis empírico, en lugar del método de Event History Analisys, porque la encuesta citada no permitía la aplicación de tal método. Además, el objetivo del artículo no consiste en estudiar la fecundidad de las generaciones a través del tiempo histórico (cohorte) ${ }^{6}$, sino en el análisis transversal de los diferentes factores que mejor predicen la probabilidad de que se tengan hijos en España

5. Para más información sobre la encuesta y el cuestionario de la misma, ver $<\mathrm{http}$ ://www.segcial.es/prdi00/groups/public/documents/binario/097551.pdf >, donde se encuentra detallado el cuestionario y los aspectos más técnicos de la encuesta.

6. Este análisis no tiene como prioridad el estudio exclusivo de la fecundidad, sino utilizar este indicador como un elemento más de los procesos de cambio familiar producidos en España en un momento concreto del tiempo. En cualquier caso, la metodología aplicada para analizar la reproducción no está exenta de dificultades, tanto en lo que se refiere a los valores de la fecundidad del momento, como a la fecundidad de las generaciones (Devolder y Cabré, 2009). Reitero que no ha sido el objetivo y, por tanto, asumiendo las limitaciones de este análisis, que no ha tenido en cuenta factores como la edad de la maternidad, el esparcimiento entre los nacimientos y la generación, se ha tratado de analizar qué factores predicen mejor el hecho de que en España se tengan hijos. 
y de que la mujer esté empleada. Si bien la aplicación de este método tiene alguna limitación, también es cierto que permite establecer asociaciones entre variables que son de gran utilidad para estimar tendencias que, de otra forma, no podrían obtenerse.

En cuanto al primer objetivo, se definió un paquete de variables independientes (edad, estado civil, nivel de estudios del varón y de la mujer, situación laboral de ambos, estatus ocupacional de ambos, tipo de trabajo, miembro de la pareja que aporta más ingresos, ayudas económicas recibidas por hijo, ingresos familiares, dificultad para compatibilizar trabajo y familia y cooperación del compañero en las tareas domésticas), de las cuales, y tras aplicar varios modelos de regresión para obtener el más adecuado, sólo unas cuantas resultaron ser relevantes para nuestro objeto de estudio (edad, estado civil, nivel de estudios de la mujer, varón como principal sustentador económico y situación laboral de la mujer).

En el segundo objetivo propuesto, relativo a los factores que mejor predicen el empleo femenino en España, se ha seguido la misma estrategia metodológica que en el análisis anterior. Una vez definida la variable dependiente (situación laboral de la mujer), se definió un paquete de variables independientes que, a priori, se consideró que podrían influir en la situación laboral de las mujeres. Dichas variables eran las siguientes: la edad, el nivel de estudios de los miembros de la pareja, el número y la edad de los hijos, el estatus ocupacional del compañero, los ingresos del hogar, las ayudas económicas recibidas por hijo, la dificultad para compatibilizar trabajo y familia y la cooperación del compañero en las tareas domésticas. Este análisis se basa en explicar la situación laboral de la mujer. Se parte de la hipótesis de que la probabilidad de que la mujer trabaje en España estaría relacionada con el nivel educativo y la situación familiar (estado civil, número de hijos en el hogar, etc.). Al igual que en el caso anterior, se probaron numerosos modelos de análisis hasta que se obtuvo uno que se consideró satisfactorio. El mejor modelo que se ha podido estimar a partir de la muestra de datos incluía sólo las variables relativas al número de hijos, la edad, el estado civil y el nivel de estudios.

\section{Tipologías familiares, empleo femenino y reproducción en perspectiva comparada europea}

La crisis y el declive de las denominadas «familias de un solo sustentador varón» es un hecho claramente documentado a nivel europeo en numerosas investigaciones (Lewis, 1992; Pfau-Effinger, 2005). Incluso la relativa persistencia de este modelo familiar en los países del sur de Europa (Blossfeld y Drobnic, 2001) da muestras evidentes de un lento declive de este tipo de familias, aunque con cierto retraso (Naldini, 2003; Moreno Mínguez, 2007). De hecho, los datos parecen confirmar que en España se está produciendo una erosión del modelo de varón sustentador, aunque en menor medida que en otros países europeos. En el gráfico 1, se constata el hecho de que en España el 39,5\% de las familias están formadas por un solo sustentador, generalmente varón, frente 

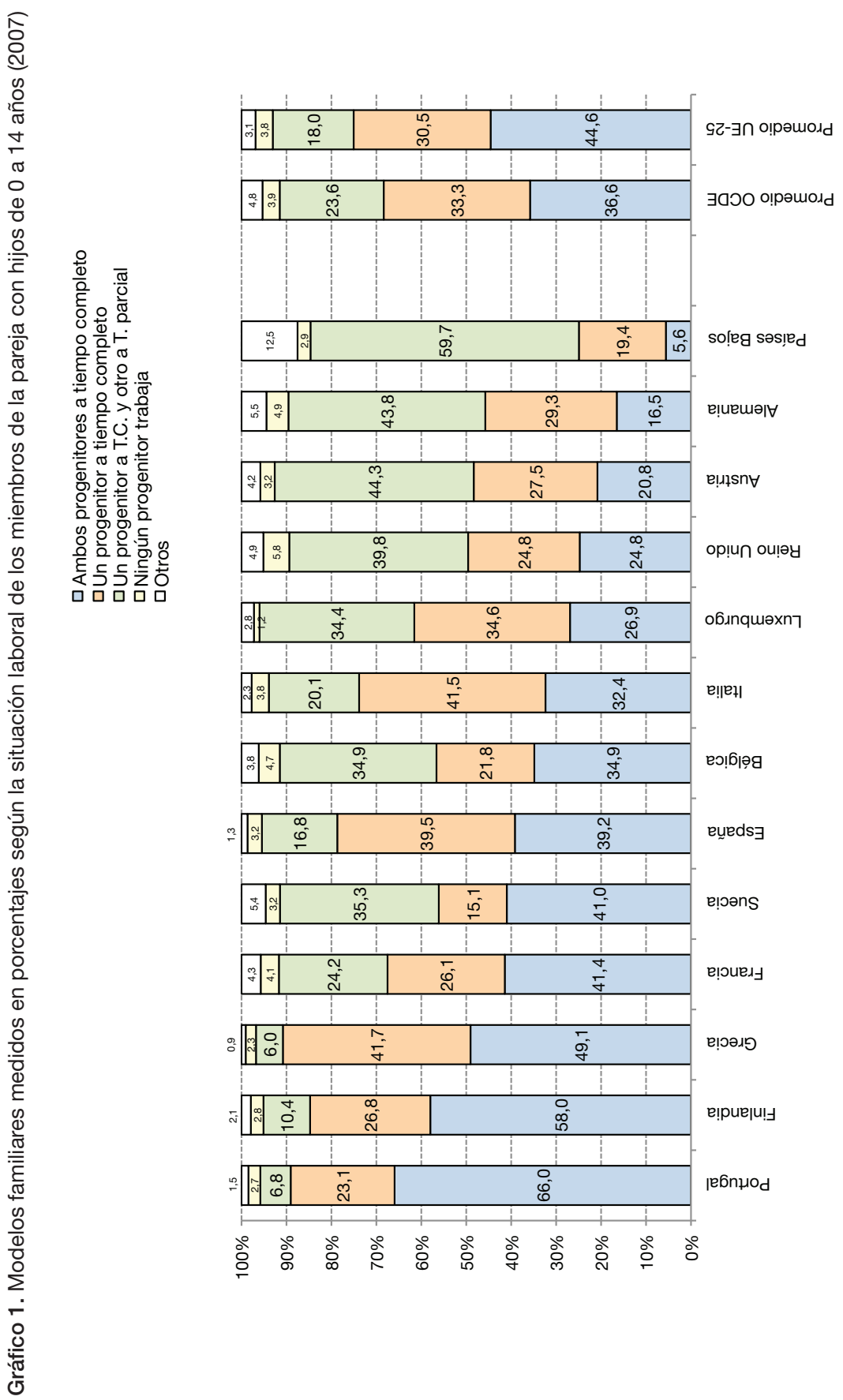
Gráfico 2. Evolución expresada en porcentajes de los modelos familiares según la situación laboral de la pareja con o sin hijos (2000-2006)

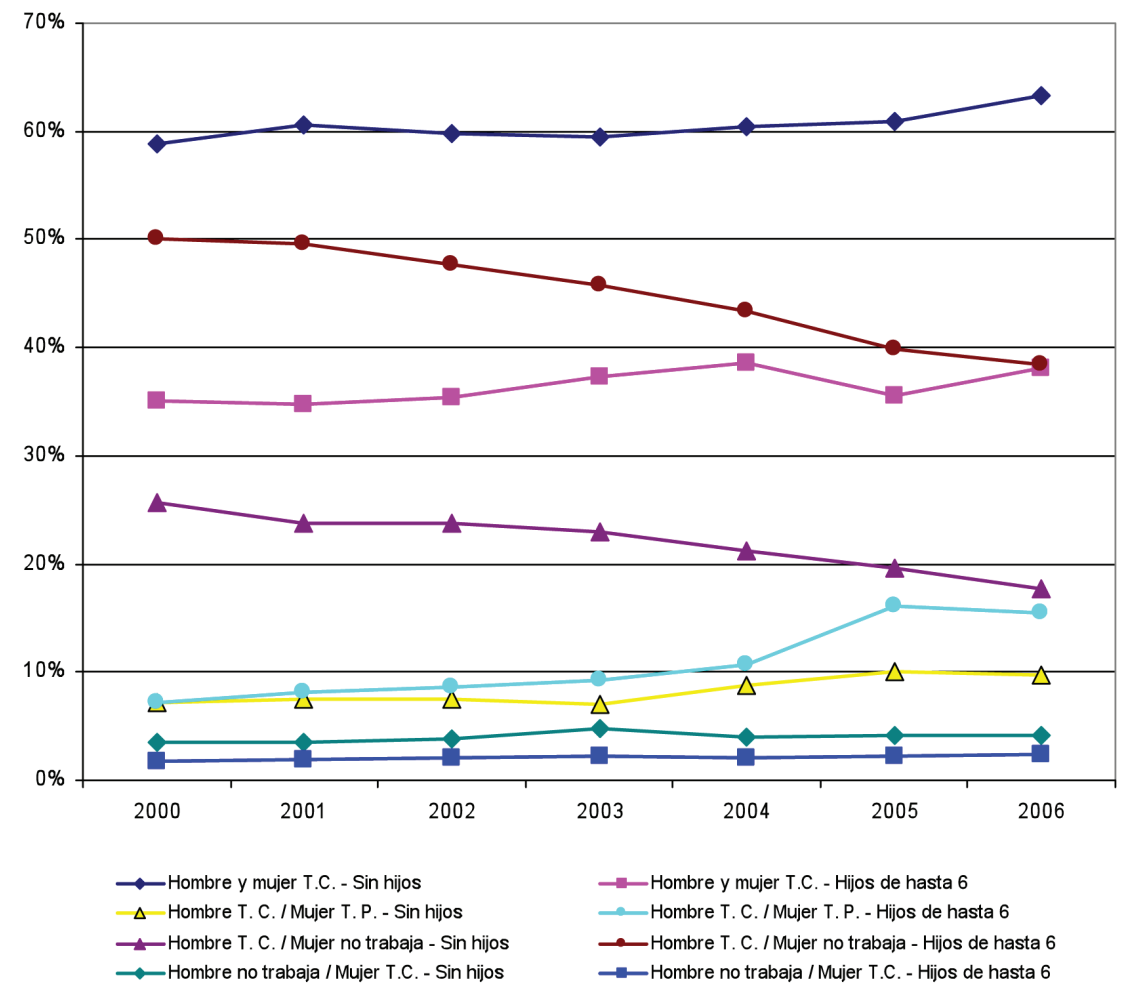

Fuente: elaboración propia a partir de los microdatos de la European Labour Survey, 2000-2006.

al 26,8\% que representa este tipo de familia en Finlandia o el 15,1\% en Suecia. Destaca también en esta tabla el modelo de hogar en el que uno de sus miembros trabaja a tiempo parcial. Este modelo familiar y laboral es una estrategia extendida en Países Bajos (59,7\%), Austria (44,3\%) y Alemania (43,8\%). Tal y como apunta Ranci (2010: 37), esto no significa, para el caso de los países del sur de Europa, que el modelo de organización familiar basada en la división tradicional del trabajo y del cuidado esté desapareciendo. Se trata más bien de una transformación que de una desaparición; transformación familiar que está estrechamente relacionada con las pautas reproductivas.

Los datos del gráfico 2, referidos a España, destacan el hecho de que las familias de dos sustentadores son más numerosas entre las parejas sin hijos que entre las parejas con hijos. Se aprecia también el relativo incremento que se ha producido en las familias de dos sustentadores, fundamentalmente, entre las parejas sin hijos desde el año 2000, aunque también cabe destacar el ligero aumento de este tipo de familias entre las parejas con hijos de hasta seis años. 
Los datos permiten constatar, por tanto, la erosión del modelo familiar de un solo sustentador varón, sobre todo en las parejas sin hijos, pero también, aunque en menor medida, en las parejas con hijos. Finalmente, cabe subrayar que el dato referido al modelo familiar en el que la mujer trabaja a tiempo parcial en las parejas con hijos tiene una intensidad distinta en los diferentes países. Este hecho puede ser interpretado, dependiendo del contexto laboral y cultural, como una posible estrategia de conciliación adoptada fundamentalmente por las mujeres en contextos institucionales y laborales favorables a la incorporación femenina en el mercado laboral. En el caso de España, tal y como se puede observar en el gráfico 2, se trata de una estrategia muy poco frecuente entre las parejas con hijos, aunque en progresivo aumento desde el año 2004.

En la tabla 1, se constata el impacto negativo que tienen los hijos en el empleo femenino, tanto en lo que se refiere a la edad como al número de hijos, lo cual es especialmente destacable en países como España e Italia en comparación con Noruega, Suecia y Finlandia. En el caso de los varones, los hijos parecen tener un efecto positivo en el empleo, mientras que, para las mujeres, los hijos se correlacionan negativamente con su participación laboral. A este respecto, cabe destacar que la brecha de género es considerablemente mayor en los países del sur de Europa en comparación con Noruega y Suecia.

Un indicador que refleja también el impacto negativo de los hijos sobre el empleo femenino es el referido al número de hijos según la tipología familiar. En el caso de España, destaca el hecho de que, en los hogares con un solo sustentador (generalmente varón), la fecundidad es mayor que en los hogares con dos sustentadores económicos. Según los datos de la European Labour Survey, para España, en el año 2006, el 34\% de las familias de un solo sustentador varón tenían más de tres hijos, frente al $29 \%$ de las familias de dos sustentadores. Este dato nos informa de la incidencia que tienen los roles de género en la fecundidad y podría sustentar la hipótesis de que el modelo tradicional de familia se asocia con una mayor fecundidad, mientras que el modelo de relaciones de género en el que ambos cónyuges trabajan y supuestamente comparten las tareas del hogar se asocia con un menor número de hijos. En definitiva, estos datos están evidenciando que, en los países del sur de Europa, las familias con hijos se asocian en mayor medida que en los países del norte de Europa con el modelo de varón sustentador. Hallazgo que ya ha sido apuntado por Cooke (2001) y Ranci (2009) en sus trabajos respectivos.

En relación con este indicador, destaca el hecho de que en todos los países de referencia el porcentaje de mujeres con más de dos hijos es mayor entre las denominadas «amas de casa» que entre las mujeres que trabajan, pero este porcentaje es especialmente elevado en España e Italia comparado con Noruega o Suecia (ver los gráficos 3 y 4 ).

En este complejo marco de asociaciones entre el empleo femenino y la maternidad, en el que resulta difícil determinar la incidencia de los diferentes factores intervinientes, la educación es uno de los factores que mejor explica las variaciones producidas, tanto en el empleo femenino como en la fecundidad en España, según los diferentes estudios realizados. Los datos comparados de 
Tabla 1. Tasa de empleo por sexo, edad de los hijos y número de hijos (año 2007)

\begin{tabular}{|c|c|c|c|c|c|c|c|c|c|c|}
\hline & & \multirow[b]{2}{*}{ TOTAL } & \multicolumn{3}{|c|}{ Edad del hijo menor } & \multicolumn{5}{|c|}{ Número de hijos menores de 18 años } \\
\hline & & & Sin hijos & $\begin{array}{c}\text { Menor de } \\
6 \text { años }\end{array}$ & $\begin{array}{c}\text { De } 6 \text { a } \\
17 \text { años }\end{array}$ & Sin hijos & 1 hijo & 2 hijos & 3 hijos & $\begin{array}{l}4 \text { hijos } \\
\text { o más }\end{array}$ \\
\hline \multirow[t]{2}{*}{ Austria } & Varones & $91,9 \%$ & $87,7 \%$ & $98,0 \%$ & $95,5 \%$ & $87,7 \%$ & $96,1 \%$ & $97,5 \%$ & & \\
\hline & Mujeres & $66,3 \%$ & $74,7 \%$ & $42,4 \%$ & $69,5 \%$ & $74,7 \%$ & $66,3 \%$ & $53,7 \%$ & & \\
\hline \multirow[t]{2}{*}{ Bélgica } & Varones & $86,5 \%$ & $81,1 \%$ & $93,3 \%$ & $90,5 \%$ & $81,1 \%$ & $90,6 \%$ & $94,1 \%$ & $86,7 \%$ & \\
\hline & Mujeres & $67,3 \%$ & $65,1 \%$ & $70,3 \%$ & $68,6 \%$ & $65,1 \%$ & $68,5 \%$ & $73,6 \%$ & $66,9 \%$ & \\
\hline \multirow[t]{2}{*}{ Alemania } & Varones & $88,0 \%$ & $85,0 \%$ & $93,3 \%$ & $91,5 \%$ & $85,0 \%$ & $90,7 \%$ & $94,7 \%$ & $89,9 \%$ & \\
\hline & Mujeres & $68,2 \%$ & $78,1 \%$ & $31,6 \%$ & $70,0 \%$ & $78,1 \%$ & $64,8 \%$ & $49,6 \%$ & $36,1 \%$ & \\
\hline \multirow[t]{2}{*}{ Dinamarca } & Varones & $84,4 \%$ & $79,2 \%$ & $90,9 \%$ & $92,3 \%$ & $79,2 \%$ & $90,6 \%$ & $93,4 \%$ & $91,9 \%$ & $78,9 \%$ \\
\hline & Mujeres & $78,0 \%$ & $75,9 \%$ & $75,9 \%$ & $83,8 \%$ & $75,9 \%$ & $78,9 \%$ & $83,4 \%$ & $77,8 \%$ & $57,9 \%$ \\
\hline \multirow[t]{2}{*}{ España } & Varones & $84,0 \%$ & $80,1 \%$ & $94,7 \%$ & $84,2 \%$ & $80,1 \%$ & $84,8 \%$ & $93,2 \%$ & & \\
\hline & Mujeres & $48,0 \%$ & $54,0 \%$ & $43,7 \%$ & $40,8 \%$ & $54,0 \%$ & $43,9 \%$ & $40,0 \%$ & $38,2 \%$ & \\
\hline \multirow[t]{2}{*}{ Finlandia } & Varones & $78,9 \%$ & $71,8 \%$ & $89,3 \%$ & $88,5 \%$ & $71,8 \%$ & $86,5 \%$ & $91,7 \%$ & $88,6 \%$ & $86,1 \%$ \\
\hline & Mujeres & $68,3 \%$ & $70,7 \%$ & $45,1 \%$ & $80,9 \%$ & $70,7 \%$ & $67,2 \%$ & $70,3 \%$ & $57,9 \%$ & $44,4 \%$ \\
\hline \multirow[t]{2}{*}{ Grecia } & Varones & $86,8 \%$ & $80,6 \%$ & $96,2 \%$ & $92,1 \%$ & $80,6 \%$ & $89,6 \%$ & $97,1 \%$ & $94,3 \%$ & \\
\hline & Mujeres & $49,6 \%$ & $49,1 \%$ & $48,8 \%$ & $50,7 \%$ & $49,1 \%$ & $51,6 \%$ & $50,4 \%$ & $41,4 \%$ & \\
\hline \multirow[t]{2}{*}{ Irlanda } & Varones & $84,3 \%$ & $78,4 \%$ & $92,7 \%$ & $86,7 \%$ & $78,4 \%$ & $86,6 \%$ & $92,4 \%$ & $89,3 \%$ & $88,9 \%$ \\
\hline & Mujeres & $57,9 \%$ & $67,6 \%$ & $52,9 \%$ & $51,3 \%$ & $67,6 \%$ & $59,0 \%$ & $49,1 \%$ & $51,4 \%$ & $35,1 \%$ \\
\hline \multirow[t]{2}{*}{ Italia } & Varones & $81,0 \%$ & $74,1 \%$ & $93,2 \%$ & $86,7 \%$ & $74,1 \%$ & $86,5 \%$ & $92,9 \%$ & $88,1 \%$ & \\
\hline & Mujeres & $50,1 \%$ & $52,2 \%$ & $48,7 \%$ & $47,4 \%$ & $52,2 \%$ & $50,5 \%$ & $47,0 \%$ & $34,6 \%$ & \\
\hline \multirow[t]{2}{*}{ Noruega } & Varones & $86,0 \%$ & $80,3 \%$ & $95,1 \%$ & $91,4 \%$ & $80,3 \%$ & $90,3 \%$ & $95,2 \%$ & $95,3 \%$ & $92,0 \%$ \\
\hline & Mujeres & $77,2 \%$ & $74,5 \%$ & $75,0 \%$ & $83,8 \%$ & $74,5 \%$ & $80,8 \%$ & $81,3 \%$ & $76,7 \%$ & $59,6 \%$ \\
\hline \multirow[t]{2}{*}{ Suecia } & Varones & $93,3 \%$ & $90,4 \%$ & $97,5 \%$ & $96,5 \%$ & $90,4 \%$ & $96,0 \%$ & $97,6 \%$ & $97,3 \%$ & $97,2 \%$ \\
\hline & Mujeres & $91,1 \%$ & $88,9 \%$ & $92,2 \%$ & $93,8 \%$ & $88,9 \%$ & $91,7 \%$ & $95,0 \%$ & $93,1 \%$ & $88,4 \%$ \\
\hline \multirow[t]{2}{*}{ Taiwan } & Varones & $86,4 \%$ & $78,2 \%$ & $95,0 \%$ & $90,8 \%$ & $78,2 \%$ & $87,5 \%$ & $95,1 \%$ & $95,7 \%$ & $97,2 \%$ \\
\hline & Mujeres & $60,5 \%$ & $61,9 \%$ & $54,2 \%$ & $63,1 \%$ & $61,9 \%$ & $61,0 \%$ & $59,4 \%$ & $57,8 \%$ & $53,0 \%$ \\
\hline \multirow[t]{2}{*}{ EE.UU. } & Varones & $85,2 \%$ & $81,7 \%$ & $90,6 \%$ & $87,8 \%$ & $81,7 \%$ & $86,7 \%$ & $91,3 \%$ & $91,5 \%$ & $86,3 \%$ \\
\hline & Mujeres & $70,4 \%$ & $76,4 \%$ & $57,6 \%$ & $72,0 \%$ & $76,4 \%$ & $71,0 \%$ & $65,3 \%$ & $58,9 \%$ & $46,4 \%$ \\
\hline
\end{tabular}

Fuente: elaboración propia a partir de la base de datos LIS, 2009.

la tabla 2 constatan la asociación negativa entre formación y número de hijos para España, en comparación con Suecia y Finlandia. Esta asociación negativa es más significativa en el caso de las mujeres que de los varones. Estos datos, avalados por numerosos estudios empíricos, nos permiten subrayar que, a nivel macro, la educación superior de la mujer incide negativamente, en cierta forma, en el número de hijos en España e Italia, mientras que en Noruega y Suecia 
Gráfico 3. Número de hijos tenidos por las mujeres amas de casa de 15 a 55 años según país (en tantos por ciento)

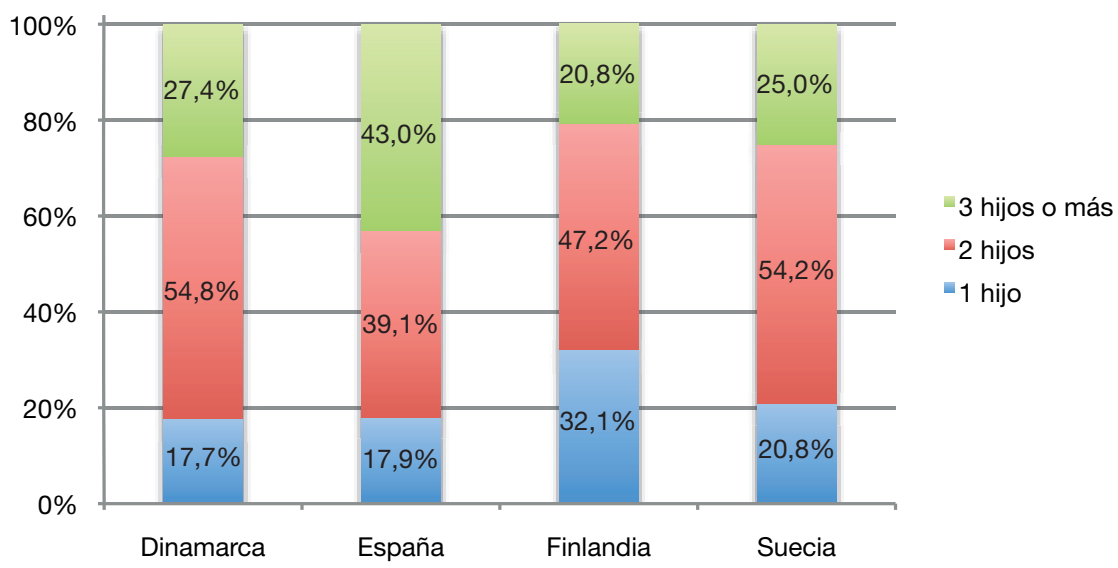

Fuente: elaboración propia a partir de los datos de la European Social Survey, 2006.

Gráfico 4. Número de hijos tenidos por las mujeres trabajadoras de 15 a 55 años según país (en tantos por ciento)

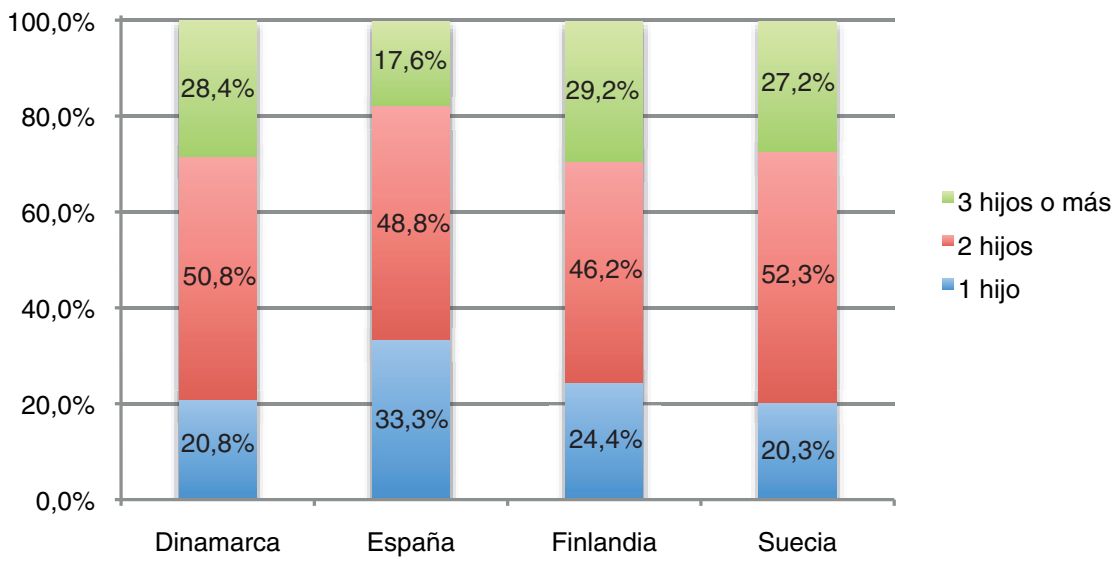

Fuente: elaboración propia a partir de los datos de la European Social Survey, 2006. 
Tabla 2. Correlaciones de Pearson entre el número de hijos y la formación de los padres

\begin{tabular}{lcccc}
\hline & España & Suecia & Finlandia & Todos los países \\
\hline Formación de la madre & $-0,291$ & $-0,083$ & $-0,164$ & 0,221 \\
Fomación del padre & $-0,123$ & $-0,035$ & $-0,047$ & 0,185 \\
\hline
\end{tabular}

Notas: Nivel de significación 0,001 0,01 0,05

Fuente: elaboración propia a partir de los datos de la European Social Survey, 2006.

las diferencias por nivel educativo y número de hijos son menos significativas. Estos datos también apuntan indirectamente al hecho de que un contexto institucional más favorable para la compatibilización laboral y familiar, como en el caso de Suecia y Noruega, puede contribuir a minimizar el impacto negativo que tiene la formación de la mujer sobre la fecundidad en contextos de limitado apoyo a las familias con dos sustentadores. En el caso de los varones, la formación tiene menor relevancia para explicar las diferencias en el número de hijos que en el caso de las mujeres. Esto podría estar evidenciando la desigualdad de género existente en torno a la maternidad y la paternidad respectivamente, ya que, según estos datos, los hijos suponen un mayor coste para las mujeres con educación superior que para los varones con el mismo nivel educativo. En cualquier caso, estos resultados hay que interpretarlos con cierta cautela, ya que se trata de un análisis transversal del momento, y estos procesos cambian a lo largo del ciclo vital de los individuos.

La tabla 3 recoge los resultados del análisis de regresión realizado a partir de los datos de la European Labour Survey 2006 para explicar la probabilidad del modelo familiar de varón y sustentador y el modelo familiar de dos sustentadores en los diferentes países europeos para los que hay datos disponibles.

En términos generales, las variables con más importancia en los dos análisis son el nivel de educación de la mujer y el número de hijos de 0 a 4 años. Las probabilidades de que un hogar tenga un solo sustentador varón disminuyen a medida que el nivel educativo de la mujer es más elevado y aumentan con el número de hijos de 0 a 4 años. Por el contrario, las probabilidades de que en un hogar haya dos sustentadores aumentan a medida que sube el nivel de educación de la mujer y disminuyen con el número de hijos de 0 a 4 años. Por países, la variable referida al número de hijos menores de 4 años es la que mejor explica la probabilidad de que el hogar familiar tenga un solo sustentador varón, seguida de la formación de la mujer en países como Austria, Alemania y Reino Unido. Es decir, en estos países, a mayor número de hijos entre 0 y 4 años y a menor nivel educativo de la mujer, mayor es la probabilidad de que en el hogar familiar haya un solo sustentador varón. En países como Bélgica, Italia y España, la educación de la mujer seguida del número de hijos entre 0 y 4 años son las variables que mejor predicen la probabilidad de que haya un hogar con un sustentador económico o dos sustentadores.

El nivel educativo del hombre es un factor explicativo en Francia, Portugal, Países Bajos, Luxemburgo, Reino Unido y España. Los índices constatan 
Tabla 3. Probabilidad de que haya una familia con un sustentador económico varón y con dos sustentadores económicos. Datos del año 2006 clasificados por países

\begin{tabular}{|c|c|c|c|c|c|c|c|c|}
\hline & \multicolumn{2}{|c|}{ Austria } & \multicolumn{2}{|c|}{ Bélgica } & \multicolumn{2}{|c|}{ Alemania } & \multicolumn{2}{|c|}{ España } \\
\hline & $\begin{array}{l}\text { Un sustentador } \\
\text { varón }\end{array}$ & $\begin{array}{c}\text { Dos } \\
\text { sustentadores }\end{array}$ & $\begin{array}{l}\text { Un sustentador } \\
\text { varón }\end{array}$ & $\begin{array}{c}\text { Dos } \\
\text { sustentadores }\end{array}$ & $\begin{array}{l}\text { Un sustentador } \\
\text { varón }\end{array}$ & $\begin{array}{c}\text { Dos } \\
\text { sustentadores }\end{array}$ & $\begin{array}{l}\text { Un sustentador } \\
\text { varón }\end{array}$ & $\begin{array}{c}\text { Dos } \\
\text { sustentadores }\end{array}$ \\
\hline \multicolumn{9}{|l|}{ Hijos de 0 a 4 años } \\
\hline \multicolumn{9}{|l|}{ Ninguno } \\
\hline Uno & 2,296 & 0,436 & 2,218 & 0,451 & 3,724 & 0,269 & 1,449 & 0,690 \\
\hline Dos o más & 7,049 & 0,142 & 2,788 & 0,359 & 9,232 & 0,108 & 2,155 & 0,464 \\
\hline \multicolumn{9}{|l|}{ Hijos de 5 a 9 años } \\
\hline \multicolumn{9}{|l|}{ Ninguno } \\
\hline Uno & 1,604 & 0,623 & & & 1,463 & 0,684 & 1,301 & 0,768 \\
\hline Dos o más & 2,888 & 0,346 & 1,834 & 0,545 & 2,382 & 0,420 & 1,563 & 0,640 \\
\hline \multicolumn{9}{|l|}{ Hijos de 10 a 14 años } \\
\hline \multicolumn{9}{|l|}{ Ninguno } \\
\hline Uno & 1,251 & 0,799 & 1,408 & 0,710 & 1,350 & 0,741 & & \\
\hline Dos o más & 1,526 & 0,655 & 1,793 & 0,558 & 1,642 & 0,609 & & \\
\hline \multicolumn{9}{|c|}{$\begin{array}{l}\text { Cuidar niños: razón para no trabajar } \\
\text { (mujer) } \\
\text { Carencia de guarderias }\end{array}$} \\
\hline \multicolumn{9}{|l|}{ Coste de guarderías } \\
\hline \multicolumn{9}{|l|}{ Baja calidad guarderías } \\
\hline Otras razones & 2,881 & 0,347 & & & 6,837 & 0,146 & 0,635 & 1,575 \\
\hline \multicolumn{9}{|l|}{ Edad del varón } \\
\hline \multicolumn{9}{|l|}{$20-29$} \\
\hline 30-39 & & & 0,671 & 1,490 & & & & \\
\hline 40-49 & & & & & & & 1,379 & 0,725 \\
\hline \multicolumn{9}{|l|}{ Edad de la mujer } \\
\hline \multicolumn{9}{|l|}{$20-29$} \\
\hline 30-39 & 0,729 & 1,372 & & & 0,655 & 1,527 & & \\
\hline $40-49$ & & & & & 0,685 & 1,461 & & \\
\hline \multicolumn{9}{|l|}{ Edad del varón } \\
\hline \multicolumn{9}{|l|}{ Baja } \\
\hline Media & & & & & & & 0,899 & 1,112 \\
\hline \multicolumn{9}{|l|}{ Alta } \\
\hline \multicolumn{9}{|l|}{ Formación de la mujer } \\
\hline \multicolumn{9}{|l|}{ Baja (estudios primarios) } \\
\hline Media (estudios secundarios) & 0,417 & 2,395 & 0,401 & 2,494 & 0,412 & 2,429 & 0,480 & 2,085 \\
\hline Alta (estudios superiores) & 0,245 & 4,077 & 0,147 & 6,822 & 0,228 & 4,386 & 0,228 & 4,385 \\
\hline Ocupación del marido & & & & & & & & \\
\hline Directivo y ejecutivo & & & & & & & & \\
\hline Científico e intelectual & & & & & & & 0,800 & 1,251 \\
\hline Técnico de nivel medio & & & & & & & & \\
\hline Empleado de oficina & & & & & & & & \\
\hline Trabajador de servicios y vend & & & & & & & & \\
\hline Agricultor y técnico calificado & 0,433 & 2,310 & & & & & & \\
\hline Oficial, operario y artesano & & & & & & & 1,375 & 0,727 \\
\hline Operador de instalaciones... & & & & & & & 1,288 & 0,776 \\
\hline Trabajador no cualificados & & & & & & & & \\
\hline R2 de Nagelkerke & 0,186 & 0,186 & 0,154 & 0,154 & 0,218 & 0,218 & 0,224 & 0,224 \\
\hline N (tamaño de la muestra) & 3.749 & 3.749 & 2.310 & 2.310 & 3.711 & 3.711 & 13.832 & 13.832 \\
\hline
\end{tabular}

Nota: Se han inlcuido únicamente las ratios que son significativas al $10 \%$, al $5 \%$ y al $1 \%$, respectivamente.

Fuente: elaboración propia a partir de los datos de la European Labor Force Survey, 2006. 


\begin{tabular}{|c|c|c|c|c|c|c|c|c|c|}
\hline \multicolumn{2}{|c|}{ Francia } & \multicolumn{2}{|c|}{ Grecia } & \multicolumn{2}{|c|}{ Italia } & \multicolumn{2}{|c|}{ Paises Bajos } & \multicolumn{2}{|c|}{ Reino Unido } \\
\hline $\begin{array}{l}\text { Un sustentador } \\
\text { varón }\end{array}$ & $\begin{array}{c}\text { Dos } \\
\text { sustentadores }\end{array}$ & $\begin{array}{l}\text { Un sustentador } \\
\text { varón }\end{array}$ & $\begin{array}{c}\text { Dos } \\
\text { sustentadores }\end{array}$ & $\begin{array}{l}\text { Un sustentador } \\
\text { varón }\end{array}$ & $\begin{array}{c}\text { Dos } \\
\text { sustentadores }\end{array}$ & $\begin{array}{l}\text { Un sustentador } \\
\text { varón }\end{array}$ & $\begin{array}{c}\text { Dos } \\
\text { sustentadores }\end{array}$ & $\begin{array}{l}\text { Un sustentador } \\
\text { varón }\end{array}$ & $\begin{array}{c}\text { Dos } \\
\text { sustentadores }\end{array}$ \\
\hline & & 1,510 & 0,662 & 1,549 & 0,645 & 1,570 & 0,637 & 3,001 & 0,333 \\
\hline & & 2,281 & 0,438 & 2,724 & 0,367 & 1,972 & 0,507 & 7,077 & 0,378 \\
\hline & & 1,232 & 0,812 & 1,270 & 0,787 & 1,423 & 0,703 & 1,555 & 0,643 \\
\hline & & 1,481 & 0,675 & 1,950 & 0,513 & 1,666 & 0,600 & 2,648 & 0,378 \\
\hline & & & & 1,379 & 0,725 & & & 1,433 & 0,698 \\
\hline & & & & 2,167 & 0,461 & & & 1,964 & 0,509 \\
\hline \multirow{4}{*}{0,436} & & & & & & 4,837 & 0,207 & & \\
\hline & & & & & & 11,379 & 0,088 & & \\
\hline & 2,295 & & & & & 3,084 & 0,324 & & \\
\hline & & 0,672 & 1,488 & 0,852 & 1,174 & & & 0,852 & 1,174 \\
\hline 0,620 & 1,614 & 0,782 & 1,279 & 0,561 & 1,783 & 0,780 & 1,128 & 0,636 & 1,573 \\
\hline \multirow[t]{2}{*}{0,484} & 2,065 & 0,780 & 1,282 & 0,545 & 1,835 & & & 0,654 & 1,529 \\
\hline & & & & & & & & 1,172 & 0,853 \\
\hline 1,597 & 0,626 & & & & & 1,362 & 0,734 & 1,357 & 0,737 \\
\hline 0,531 & 1,884 & 0,638 & 1,568 & 0,386 & 2,591 & 0,409 & 2,444 & 0,612 & 1,635 \\
\hline 0,282 & 3,540 & 0,179 & 5,582 & 0,150 & 6,655 & 0,229 & 4,359 & 0,319 & 3,136 \\
\hline & & & & 0,791 & 1,264 & & & & \\
\hline 0,662 & 1,511 & 1,531 & 0,653 & 0,710 & 1,408 & & & 0,786 & 1,272 \\
\hline \multirow[t]{5}{*}{0,537} & 1,862 & & & & & & & & \\
\hline & & 0,617 & 1,622 & & & & & & \\
\hline & & 1,754 & 0,570 & 1,232 & 0,811 & & & 0,820 & 1,219 \\
\hline & & 1,624 & 0,616 & & & 1,416 & 0,706 & & \\
\hline & & 1,446 & 0,692 & 1,534 & 0,652 & & & & \\
\hline 0,237 & 0,237 & 0,151 & 0,151 & 0,175 & 0,175 & 0,155 & 0,155 & 0,153 & 0,153 \\
\hline 3.245 & 3.245 & 7.114 & 7.114 & 15.075 & 15.075 & 7.725 & 7.725 & 9.428 & 9.428 \\
\hline
\end{tabular}


que, en los cinco primeros países, la probabilidad de que el hogar tenga dos sustentadores económicos desciende a medida que aumenta el nivel educativo del varón. En el caso español, la probabilidad de que el hogar tenga un solo sustentador desciende si el hombre tiene un nivel de estudios medios frente al nivel educativo más bajo, mientras la probabilidad de que el hogar tenga dos sustentadores económicos aumenta si el hombre tiene estudios medios.

En cuanto al resto de las variables, destaca el hecho de que las razones dadas por las mujeres para no trabajar atribuibles al cuidado de los niños es otro de los factores que explica el hecho de que el hogar familiar tenga un solo sustentador o dos sustentadores económicos. En Francia y en los Países Bajos es el primer factor, mientras que en España es el segundo.

Destaca el hecho de que, en los Países Bajos, la probabilidad de que el varón sea el único sustentador del hogar aumenta de forma significativa si la razón por la que la mujer no trabaja no depende tanto de la carencia de servicios para el cuidado de los niños, tales como las escuelas de educación infantil y guarderías, sino más bien de la calidad y el precio de los mismos. De la misma forma, la probabilidad de que haya un hogar familiar con dos sustentadores económicos desciende si la razón aludida por la mujer para no trabajar es la reducida calidad de los servicios existentes para el cuidado de los niños.

La ocupación del varón es un factor explicativo en Polonia, Portugal, Rumanía, Irlanda, Grecia, Italia, Bulgaria, Eslovaquia, Austria, República Checa, España, Países Bajos, Reino Unido y Hungría, con resultados muy dispares. En el caso español, la probabilidad de que se dé la situación de un hogar con dos sustentadores económicos aumenta si el marido tiene una ocupación de estatus medio y alto, aunque no con valores especialmente significativos.

Finalmente, la edad de los miembros de la pareja es un factor con una incidencia desigual. Por lo general, destaca el hecho de que, a mayor edad de los miembros de la pareja, mayor es la probabilidad de encontrar un hogar con un solo sustentador varón, sobre todo en países como España, donde la incorporación masiva de la mujer al mercado laboral se ha realizado sobre todo a partir de la década de 1980.

\section{Análisis de los factores explicativos del empleo femenino y la reproducción en España en un contexto de cambio familiar}

\section{Fecundidad y cambio familiar en España}

Los resultados obtenidos para España a partir del modelo de regresión aplicado con los datos procedentes de la Encuesta de Familia y Empleo 2006 hay que interpretarlos en el contexto en el que se ha producido la evolución de la fecundidad y el empleo femenino en España. Estos dos indicadores han tenido una tendencia muy diferente a la del resto de los países europeos. Mientras que, en el conjunto de los países de la Europa de los quince, el signo de la correlación entre fecundidad y actividad femenina se torna positivo a partir de los años 
ochenta, en España continuaba siendo de signo negativo (Moreno Mínguez, 2007: 103 y 205). De hecho, actualmente, aquellos países que tienen las tasas de empleo femenino más elevadas son los que también tienen los indicadores de fecundidad más altos, como son los casos de Finlandia, Suecia, Noruega y Francia. Por el contrario, los países del sur de Europa, que tienen las tasas más reducidas de empleo femenino, son también los que tienen los más reducidos índices de fecundidad. A este respecto, hay que subrayar que el tipo de ocupación desempeñado por la mujer ha sido señalado como mejor predictor que la situación laboral femenina ${ }^{7}$. Los análisis realizados han constatado que en los países del norte de Europa, así como en Francia, los efectos negativos del empleo sobre la fecundidad se han neutralizado en parte exitosamente a través, entre otros factores, de generosas políticas familiares de childcare (servicios de atención a la infancia) y de child benefit (ayudas económicas por hijo), que han reducido el coste económico y laboral que tienen los hijos en las familias, lo cual ha potenciado indirectamente la corresponsabilidad familiar entre los miembros de la pareja.

A pesar del progresivo declive del modelo de varón sustentador evidenciado en el gráfico 1, los datos parecen constatar también que en España se trata de una transformación lenta, lo que explica en parte que el empleo femenino siga teniendo un impacto destacado sobre la fecundidad. De hecho, la reducida fecundidad se explica en parte en función de factores tales como la educación y la situación laboral de la mujer. En concordancia con la literatura existente, los análisis de regresión presentados en este artículo evidencian que el nivel académico de la mujer es una variable destacada para explicar tanto la fecundidad como el empleo femenino. Efectivamente, los datos de la tabla 3 constatan que a mayor nivel de estudios de la mujer, menor probabilidad de tener hijos. Esta asociación negativa tiene múltiples interpretaciones. Según esta interpretación, las mujeres con mayores niveles educativos y, por tanto, con elevadas expectativas respecto al empleo, tienen también mayores expectativas con respecto a la igualdad de género en lo que se refiere a la compatibilización laboral y familiar y la corresponsabilidad familiar. Es decir, un contexto de reducido apoyo institucional a las madres trabajadoras y una colaboración escasa de los compañeros varones en las tareas de crianza y trabajo doméstico incide negativamente en la fecundidad del colectivo femenino con mayores niveles académicos (Cooke, 2009; Oláh, 2003; Torr y Short, 2004). Por tanto, la inversión en capital educativo parece reducir las probabilidades de tener hijos, ya que al hecho de que estos supongan un coste laboral adicional, se une

7. El trabajo de Martín García (2010: 130) ha demostrado, a través del análisis denominado «event history» que, en el caso de España, la incidencia del empleo femenino en la fecundidad (tanto en lo que se refiere al nacimiento del primer hijo como al de los siguientes) se caracteriza por las diferencias intraocupacionales de las mujeres. Según sus observaciones, el tipo de empleo es mejor predictor que la participación laboral en suma, ya que refleja las preferencias individuales respecto a la maternidad. Este factor no ha resultado ser significativo en nuestro estudio, pero es preciso subrayar que se trata de un análisis transversal del momento, lo que puede haber contribuido a neutralizar esos efectos, que sí se observan en el análisis longitudinal. 
Tabla 4. Factores determinantes de la fecundidad en España a través del modelo de regresión Logit. Hombres y mujeres de 18 a 55 años (año 2006)

\begin{tabular}{|c|c|c|c|c|}
\hline Coeficientes & Estimación & Error estándar & $z$ Valor & $\operatorname{Pr}(>|z|)$ \\
\hline (Intercept) & $-5,2668$ & 0,8175 & $-6,443$ & $1,17 \mathrm{e}-10^{* \star \star}$ \\
\hline Edad & 0,1276 & 0,0170 & 7,507 & $6,07 e-14^{* \star \star}$ \\
\hline Casado/a & 0,1063 & 0,3425 & 0,310 & 0,756271 \\
\hline Soltero/a & $-2,7641$ & 0,3499 & $-7,901$ & $2,78 \mathrm{e}-15^{* \star \star}$ \\
\hline Nivel estudios bajo (mujer) & 0,7141 & 0,2357 & 3,030 & $0,002447^{\text {** }}$ \\
\hline Familia sustentador varón & 0,8755 & 0,2421 & 3,616 & $0,000299^{\star \star \star}$ \\
\hline Mujer ocupada (dos sustentadores) & 0,7886 & 0,2892 & 2,727 & 0,006391 *夫 \\
\hline
\end{tabular}

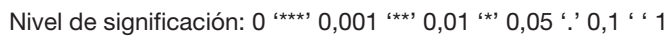

Nota: (Dispersión de los parámetros de familia binomial toma como 1). Ver gráfico 5

Desviación nula: 1060.38 en 801 grados de libertad

Desviación residual: 511,38 en 795 grados de libertad AIC: 525,38

Fuente: elaboración propia a partir de la Encuesta Familia y Empleo 2006.

la circunstancia de que la formación contribuye a aumentar las expectativas de las mujeres con respecto a la igualdad de género dentro de la familia (Bernhardt y Goldscheider, 2006). Si este proceso se produce en contextos institucionales de reducido apoyo a las madres y a los padres trabajadores, como es el caso español, los efectos negativos de la formación de la mujer sobre la fecundidad tienden a incrementarse en comparación con otros países.

Del análisis de regresión (tabla 3), resulta interesante también destacar el hecho de que la probabilidad de tener hijos se reduce en las familias de dos sustentadores, donde los dos miembros de la pareja aportan ingresos por igual, mientras que la probabilidad aumenta cuando el varón es el principal sustentador económico, hecho este último que generalmente suele estar asociado a un reparto asimétrico del trabajo doméstico entre los miembros de la pareja, según los estudios realizados al respecto. Estos resultados son acordes con los obtenidos por Cooke (2009), quien ha destacado que el reparto desigual del trabajo doméstico entre sexos predice una mayor fecundidad en los países en los que históricamente se ha potenciado una política social de apoyo a la familia donde el varón es el principal sustentador, como en Alemania. En este análisis, la renta no es un elemento determinante de la fecundidad, ya que esta variable ha sido excluida del modelo, al no ser significativa.

Por otro lado, hay que destacar que la familia tradicional, donde el varón es el único sustentador económico, está asociada generalmente, aunque no en todos los casos, con reducidos niveles educativos de los miembros de la pareja. Esta asociación ha permitido explicar a los investigadores López Blasco y Gil (2008), a través de diferentes análisis empíricos, que la fecundidad es mayor en aquellas parejas en las que la mujer tiene menor nivel educativo y donde, por tanto, prima la figura del varón sustentador entre los jóvenes españoles con edades entre los 15 y los 29 años.

A este respecto, y en un contexto de cambio acelerado en el que la mujer en España está haciendo importantes inversiones en formación y en el que se 
está produciendo una rápida transformación de los valores y de las actitudes hacia un modelo de relaciones familiares y de género más igualitario (Parella, 2008; Lück y Hofäcker, 2008), es previsible que la fecundidad pueda tender a aproximarse a los índices de los países del norte de Europa. Esto será posible si, en los próximos años, se desarrollan políticas familiares y de género que contribuyan a reducir el coste de oportunidad laboral y económico que supone tener uno o más hijos y, por tanto, a fomentar la igualdad de género, tanto en los interiores familiares como en el mercado laboral.

En relación con el argumento anterior, relativo a la persistencia de la familia tradicional y a la incidencia negativa de la educación de la mujer en la fecundidad en contextos institucionales y laborales relativamente desfavorables a la igualdad de género, no por obvio deja de ser interesante comprobar que los hijos en España se tienen a edades tardías y en el marco familiar regulado fundamentalmente a través del matrimonio, como se puede comprobar en el gráfico 5 . Se consideraron estas dos variables (edad y estado civil) para la predicción, ya que explican alrededor del $40 \%$ de la variabilidad de la probabilidad de tener hijos. Los datos sobre los nacimientos producidos fuera del matrimonio resulta interesante si se compara con otros países europeos, donde este tipo de nacimientos son una práctica extendida. El caso francés es extraordinariamente ejemplificador, ya que, según los últimos datos proporcionados por el INSEE para el año 2007, el 50,5\% de los nacidos en Francia fueron de padres que no

Gráfico 5. Predicción de la fecundidad según edad y estado civil (España 2006)

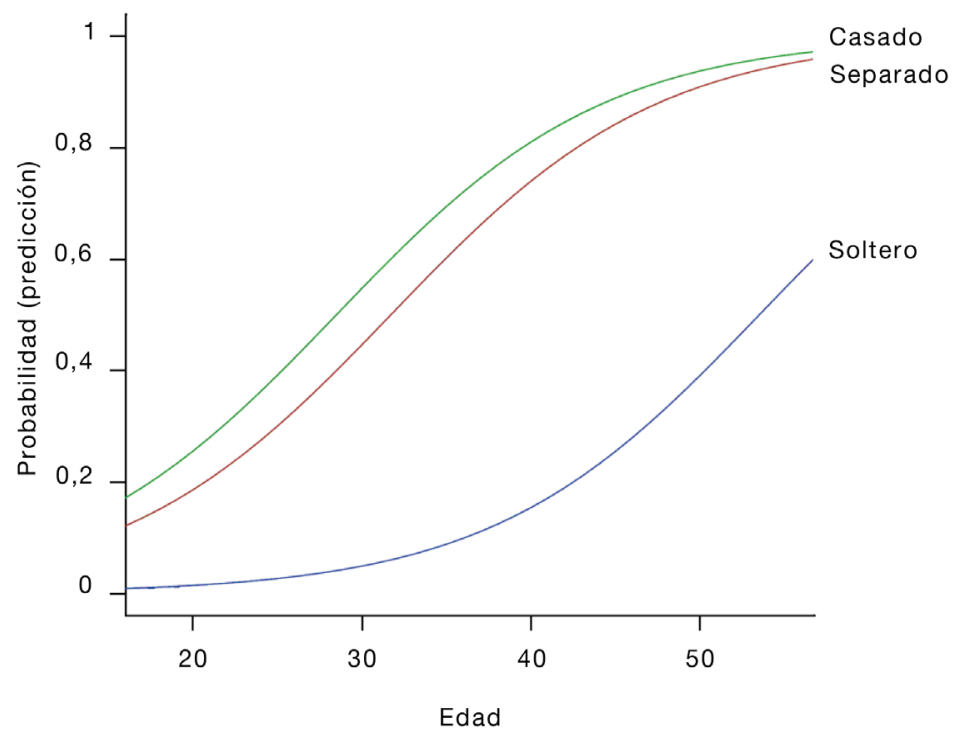

Fuente: elaboración propia a partir de la Encuesta Familia y Empleo 2006. 
estaban casados, frente al 8,5\% en 1974 y el 5,9\% en 1965. Sin embargo, para España, su peso relativo en el conjunto de la natalidad era muy reducido en 1985, puesto que suponía menos del $10 \%$ del total de nacimientos, mientras que en el año 2007 este tipo de nacimientos representaban el 30,2\% según el INE, lo que es un indicador más del progresivo cambio, pero a la vez de la persistencia de comportamientos y de formas familiares tradicionales asociadas al modelo cultural denominado familista (Moreno Mínguez, 2007).

\section{Factores determinantes del empleo femenino en España}

En lo que se refiere a los determinantes del empleo femenino, los resultados obtenidos para el caso español a partir del análisis de regresión realizado permiten constatar que los factores que mejor predicen la probabilidad de que la mujer trabaje en España son el nivel educativo y el número de hijos (ver tabla 4). El resto de las variables introducidas en el modelo no han resultado ser significativas para explicar la variable dependiente, por lo que no se han introducido en la tabla 4. Tal y como se ha puesto de manifiesto en numerosos análisis empíricos previos, en todos los países de la Europa de los quince, a mayor nivel educativo, mayor probabilidad de que la mujer trabaje. Sin embargo, en España, son especialmente significativos los efectos que tiene la educación sobre el empleo femenino, debido, entre otras razones, a las dificultades que tiene el colectivo femenino para encontrar empleo. De hecho, las diferencias en las tasas de empleo entre las mujeres por nivel educativo son más acusadas en España e Italia que, por ejemplo, en el Reino Unido o Francia (Moreno Mínguez, 2007; Michaud y Tatsiramos, 2005). El caso opuesto es Suecia, donde el nivel educativo de las mujeres apenas tiene ninguna influencia sobre el empleo femenino, ya que las condiciones del mercado de trabajo y las generosas políticas familiares dirigidas a las madres trabajadoras neutralizan en parte las diferencias laborales por nivel educativo (Gutiérrez Doménech, 2008; Gustafsson y Kalwij, 2006). Resulta paradójico comprobar que el hecho de estar casada aumenta la probabilidad de trabajar en el caso de las mujeres. Esto se debe al efecto combinado del estado civil con la edad,

Tabla 5. Factores determinantes del empleo femenino a través del modelo de regresión Logit. Mujeres de 18 a 55 años (España, 2006)

\begin{tabular}{lrcrl}
\hline \multicolumn{1}{c}{ Coeficientes } & Estimación & Error estándar & $\boldsymbol{z}$ Valor & \multicolumn{1}{c}{$\operatorname{Pr}(>|z|)$} \\
\hline (Intercept) & $-2,55432$ & 0,6421 & $-3,978$ & $6,95 \mathrm{e}-05^{* \star \star}$ \\
Casada & 2,36620 & 0,34267 & 6,905 & $5,01 \mathrm{e}-12^{* \star \star}$ \\
Soltera & 0,33917 & 0,34646 & 0,979 & 0,327595 \\
Nivel académico bajo & $-0,3453$ & 0,159 & $-2,161$ & $0,03071^{*}$ \\
\hline
\end{tabular}

Nota: hijo: spline (edad). Ver gráfico 6 para interpretación ***

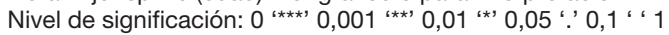

Desviación nula: 1050.68 on 801 degrees of freedom

Desviación residual: 830.14 on 791 degrees of freedom

AIC: 852.14

Fuente: encuesta Familia y Empleo 2006. 
ya que las mujeres solteras están en el proceso de búsqueda de empleo, mientras que las mujeres casadas, dada la tardía emancipación de los jóvenes en España, cuando forman una pareja estable, han consolidado o están en proceso de consolidar su situación laboral.

En el gráfico siguiente, se observa, como era de esperar, que la probabilidad de que la mujer esté empleada depende de la edad y del número de hijos. La mayor probabilidad de estar empleada se concentra en las edades intermedias, mientras que disminuye a partir de los cuarenta años. También es interesante destacar que la probabilidad de estar empleada desciende con el número de hijos, lo cual indica que este factor tiene en España un cierto efecto negativo sobre el empleo femenino, en un contexto institucional en el que no se favorece adecuadamente la compatibilización laboral y familiar. Estos datos, al igual que lo habían hecho otros estudios previos, constatan en parte la hipótesis de que las insuficientes políticas de compatibilización laboral y familiar, así como la escasez y la carestía de los servicios de atención a la infancia, junto con las precarias condiciones del mercado de trabajo, dificultan la integración de la mujer con cargas familiares en el empleo remunerado (Del Boca, 2009; Moreno Mínguez, 2007).

Los hallazgos expuestos en este estudio subrayan el hecho de que el axioma de la reducida fecundidad y el creciente, pero todavía reducido en términos comparados, empleo femenino en países como España se explica en parte en función de factores estructurales tales como la educación y la permanencia

Gráfico 6. Predicción del empleo femenino según edad y número de hijos (España 2006)

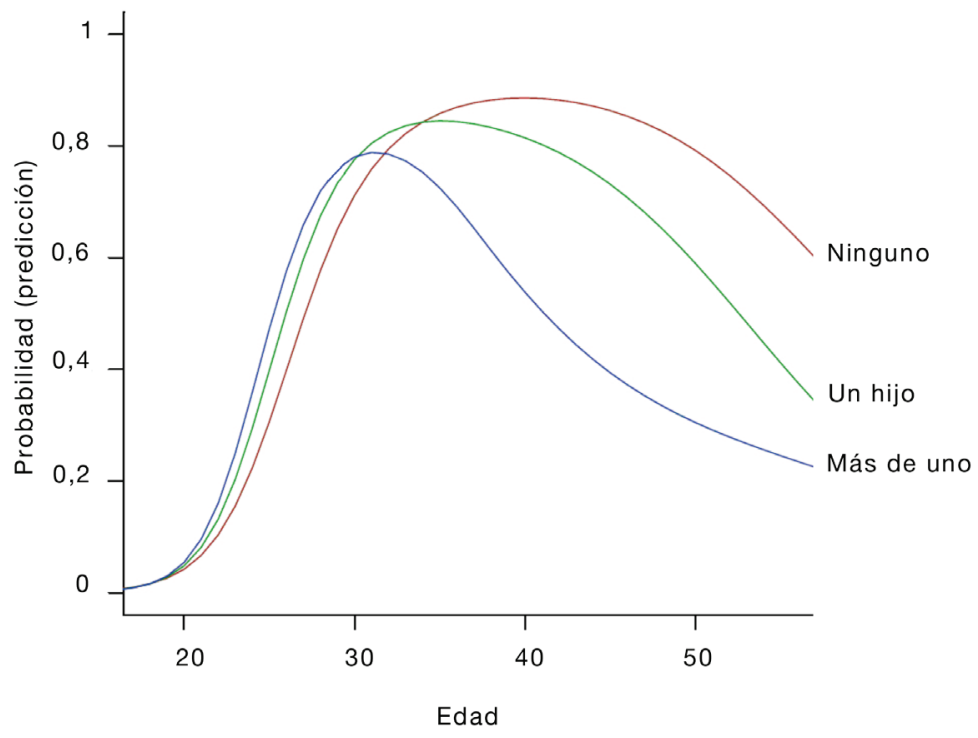

Fuente: elaboración propia a partir de la Encuesta Familia y Empleo 2006. 
de roles de género asociados con la figura del varón como principal sustentador, en un contexto institucional de poco apoyo a la compatibilización laboral y familiar. A medida que la mujer adquiere niveles educativos más elevados, sus expectativas de integrarse en el mercado de trabajo aumentan, lo que contribuye a que retrase el nacimiento del primer hijo o reduzca el número de hijos deseados, debido, entre otras razones, a que sus expectativas de emplearse no se realizan adecuadamente en unos contextos laborales, institucionales y familiares desfavorables, en que la llegada de los hijos supone para las mujeres un elevado coste de oportunidad de trabajo. En lo que se refiere al reducido empleo femenino, los análisis nos han permitido constatar que los factores de índole individual, como el nivel educativo de la mujer y el número de hijos, ejercen un relativo impacto en el empleo femenino en España, a diferencia de los países del norte de Europa, como Finlandia y Suecia, donde la incidencia del nivel educativo es menos significativo para explicar la probabilidad de que la mujer trabaje. Este hecho estaría asociado con otro hallazgo demostrado por los estudios realizados para los distintos países europeos y que también se ha puesto de manifiesto en esta investigación, que hace referencia a la reducción del impacto negativo del empleo femenino en la fecundidad y al incremento del impacto del número de hijos en el empleo femenino (Matysiak y Vignoli, 2006). Esta paradójica situación es especialmente relevante en el caso español, tal y como se ha constatado en este estudio, puesto que la edad y el número de hijos muestran una destacada incidencia negativa en la incorporación de las mujeres al mercado laboral.

\section{Conclusiones}

Los análisis presentados en este artículo han permitido constatar la complejidad de los factores que intervienen en los comportamientos de las familias ante la reproducción y el empleo femenino. Este estudio ha evidenciado que, para una adecuada compresión de los diferentes comportamientos reproductivos y laborales de los ciudadanos, y en especial de las mujeres, es necesario integrar, en los diferentes modelos explicativos, los contextos institucional, cultural y económico en los que se producen estos comportamientos, junto con factores individuales tales como la formación. Este artículo ha tratado de indagar a nivel macro los elementos de conexión existentes entre la familia (como ámbito de reproducción) y el trabajo (como ámbito de producción), en un contexto institucional, como el español, de limitado apoyo a la compatibilización laboral y familiar de los hogares con dos sustentadores económicos. Los hallazgos presentados en este artículo apuntan a que el axioma de reducida fecundidad y reducido, aunque creciente, empleo femenino, en España es el resultado de un proceso contradictorio relacionado con la lenta transformación hacia el modelo familiar de dos sustentadores económicos. Por tanto, los resultados de este artículo nos permiten concluir que, para el caso español, la transición al modelo familiar de dos sustentadores está teniendo cierto impacto negativo en la maternidad en comparación con otros países del entorno europeo. Los 
datos comparados aportados en este trabajo nos permiten destacar que, a nivel estrictamente descriptivo, para el caso español, el modelo familiar con hijos más frecuente es aquel donde el varón es el principal aunque no el único sustentador económico en comparación con otros países. De hecho, el número total de hijos es mayor en las parejas en las que la mujer no trabaja. En concordancia con este hallazgo, se observa que, en España, el empleo femenino está negativamente asociado con el número de hijos y la edad de los mismos, de modo que la brecha laboral entre hombres y mujeres a este respecto es bastante más elevada que en otros países. En relación con ello, destaca la formación de la mujer como un factor determinante generalmente asociado con el modelo familiar de dos sustentadores económicos.

Por tanto, el efecto combinado de la formación de la mujer con la tipología familiar de dos sustentadores parece tener ciertos efectos negativos en la fecundidad en comparación con otros países europeos. Esto evidencia la relevancia de otros factores intervinientes, tales como las políticas familiares de conciliación y la desigualdad de género (en el ámbito público laboral y en el privado familiar), la cultura familiar, como factores explicativos añadidos de la baja fecundidad y el reducido empleo femenino. Si bien la formación de la mujer tiene efectos positivos sobre el empleo, cuando entra en juego la variable referida a los hijos, la formación no consigue atenuar los efectos negativos que tiene la maternidad sobre el trabajo femenino, debido, entre otras razones, a las dificultades que supone compatibilizar vida laboral y familiar, así como al elevado coste personal, económico y laboral, en términos del tiempo que supone la crianza.

En lo que se refiere exclusivamente al empleo femenino, cabe destacar que la educación ejerce un efecto significativo en la probabilidad de que la mujer trabaje. Análisis empíricos comparados previos han evidenciado que esta incidencia es menor en otros países europeos, donde las mujeres tienen un contexto laboral e institucional más favorable en términos de compatibilización laboral y familiar para incorporarse al mercado laboral que en España (Moreno Mínguez, 2008). Por otro lado, los análisis realizados en este trabajo han puesto de manifiesto que el número de hijos es un factor determinante que incide negativamente en la participación laboral de las mujeres en todos los países analizados, pero especialmente en España. Por lo tanto, a partir de los resultados presentados en este trabajo, se puede concluir que, en España, el advenimiento del modelo familiar y laboral de dos sustentadores económicos está teniendo cierto efecto negativo en la fecundidad. Como síntesis, debemos subrayar que estos resultados permiten también concluir, por una parte, que los factores estructurales, tales como la formación de la mujer y el tipo de familia, tienen cierta incidencia diferencial en la fecundidad, dependiendo del contexto institucional de cada país. Por otra parte, cabe destacar también que factores tales como el número de hijos y la formación de la mujer tienen cierta incidencia en el empleo femenino en España en mayor medida que en otros países europeos. Por lo tanto, más allá de la tesis de la individualización y la pluralización de las 
preferencias familiares y laborales, los análisis presentados en este trabajo parecen apuntar a que las decisiones familiares y laborales adoptadas por los ciudadanos españoles se encuentran determinadas por un complejo mosaico de situaciones contextuales, en el que interactúan factores de tipo cultural, institucional e individual.

\section{Referencias bibliográficas}

AHN, N. y MirA, P. (2001). «Job bust, baby bust: Evidence from Spain». Journal of Population Economics, 14, 505-521.

Andersson, G.; Rønsen, M.; Knudsen, L.B.; LappegÅrd, T.; Neyer, G.; Skrede, K.; Teschner, K. y ViKat, A. (2009). "Cohort Fertility Patterns in the Nordic Countries». Demographic Research [en línea], 20 (14), 313-352. <http,//www. demographic-research.org/volumes/vol20/14/>.

AntTonen, A. y Sipilä, J. (1996). «European social care services: Is it possible to identify models?». Journal of European Social Policy, 6 (2), 87-100.

BaizÁn, P. y Martín GarCía, T. (2007). "Joint determinants of educational enrolment and first birth timing in France and West Germany». Genus, LXI (2), $89-117$.

BeCK, U. y BeCK-GernsheIm, E. (2002). Individualization, Institutionalized Individualism and its Social and Political Consequences. Londres: Sage.

Bernhardt, E. y Goldscheider, F. (2006). «Gender Equality, Parenthood Attitudes, and First Births in Sweden». Vienna Yearbook of Population Research, 2006, 19-39.

- (2008). «Domestic gender equality and childbearing: First and second births in Sweden». Conferencia presentada en Gender role division, gender role attitudes and fertility. European Population Conference. Barcelona, 9-12 de julio.

Bettio, F. y Plantenga, J. (2004). "Comparing care regimes in Europe». Feminist Economist, 10 (1), 85-113.

Billari, F. y KoHler, H.P. (2002). "Patterns of lowest-low fertility in Europe». MPIDR Working Papers WP-2002-040. Rostock: Max Planck Institute for Demographic.

BilLARI, F. y WiLSON, CH. (2001). «Convergence towards diversity?: Cohort dynamics in the transition to adulthood in contemporary Western Europe». MPIDR Working Papers WP-2001-039. Rostock: Max Planck Institute for Demographic Research.

Bittman, M.; England, P.; Folbre, N.; Sayer, L. y Matheson, G. (2003). «When Does Gender Trump Money? Bargaining and Time in Household Work». The American Journal of Sociology, 109 (1), 186-214.

Bloemen, H. y KalWIJ, A. (2001). «Female labor market transition and the timing of births, a simulthaneous analysis of the effects of schooling». Labour Economics, 8 (5), 593-620.

Blossfeld, H.P. y Drobnic, S. (eds.) (2001). Careers of Couples in Contemporary Societies: From Male Breadwinner to Dual Earner Families. Oxford: Oxford University Press.

Boje, T. y Leira, A. (2000). Gender, Welfare State and the Market: Towards a New Division of Labour. Londres: Routledge.

BRATTI, M. (2003). «Labour force participation and marital fertility of Italian women, the role of education». Journal of Population Economics, 16 (3), 525-554. 
Breen, R. y CoOKE, L. (2005). «The persistence of the gendered division of domestic labour». European Sociological Review, 21, 43-57.

Brodmann, S.; Esping-Andersen, G. y GÜELl, M. (2007). "When fertility is bargained: Second births in Denmark and Spain». European Sociological Review, 23, 599-613.

Castles, F. (2003). «The World Upside Down, Below Replacement Fertility, Changing Preferences and Family-Friendly Public Policy in 21 OECD countries». Journal of European Social Policy, 13 (3), 209-27.

Castro Martín, T.; Domínguez-Folgueras, M. y Martín-García, T. (2008). "Not trulypartnerless: Non-residential partnerships and retreat from marriage in Spain». Demographic Research [en línea], 18 (16), 443-468. <http,//www.demographicresearch.org>.

CHIURI, M.C. (2000). "Quality and demand of childcare and female labour supply in Italy». Labour, 14 (1), 97-118.

Coltrane, S. (2000). "Research on Household Labor: Modeling and Measuring the Social Embeddedness of Routine Family Work». Journal of Marriage and the Family, 62, 1208-1233.

Cooke, L.P. (2001). «Balancing Fertility and Employment in Post-Industrial Economies: A comparision of the Dual-Earner Effect on Family Size in Nine Countries». IRISS Working Paper, 2001-5.

- (2009). "Gender Equity and Fertility in Italy and Spain». Journal of Social Policy, 38 (1), 123-140.

Cordero, J. (2009). «El espaciamiento de los nacimientos, una estrategia para conciliar trabajo y familia en España». Revista Española de Investigaciones Sociológicas, 128, 11-33.

Crompton, R. (2006). Employment and the family. Cambridge: Cambridge University Press.

Crompton, R. y Lyonette, C. (2010). «Family, class and gender "strategies" in mothers' employment and childcare». En: SCOTT, J.; CROMPTON, R. y LYONETTE, C. (eds.). Gender inequalities in the 21st century: New barriers and continuing constraints. Cheltenham: Edward Elgar, 174-192.

DA Rocha, J. y Fuster, L. (2006). "Why are fertility rates and female employment ratios positively correlated across OECD countries?». International Economic Review, 47 (4), 1187-1222.

De Laat, J. y Sevilla Sanz, A. (2006). «Working Women, Men’s Home Time and Lowest-Low Fertility». ISER Working Papers 2006-23. Institute for Social and Economic Research.

Del Boca, D.; Pasqua, S. y Pronzato, C. (2004). "Why Are Fertility and Women's Employment Rates So Low in Italy?: Lessons from France and the UK». IZA Discussion Paper, 1274.

- (2009). «Motherhood and Market Work Decisions in Institutional Context: A European Perspective». Oxford Economic Papers, 61, 147-171.

Delgado, M.; De Rose, A.; Barrios, L. y Zamora, F. (2009). "The delay of maternity and its causes, an analysis of the timing of the first child in Spain». Genus, LXV (2), 79-111.

Delgado, M.; MeIl, G. y Zamora, Z. (2008). «Spain, Short on children and short on family policies». Demographic Research, 19 (27), 1059-1104.

Demeny, P. (2003). «Population policy dilemma in Europe at the dawn of the twenty first century». Population and Development Review, 29 (1), 1-28. 
Devolder, D. y Cabré, A. (2009). «Factores de la evolución de la fecundidad en España en los últimos 30 años». Panorama Social, 10, 23-39.

DunCAN, S. (2003). «Motherhood, Paid Work and Partnering: Values and Theories. Work, Employment and Society, 17 (2), 309-330.

Duvander, A-Z.; LAPPEgÅrd, T. y Andersson, G. (2010). «Family policy and fertility: fathers' and mothers' use of parental leave and continued childbearing in Norway and Sweden». Journal of European Social Policy, 20 (1), 45-57.

EsCobedo, A. (2008). «Políticas de licencias parentales y de atención infantil para los menores de tres años y sus familias: El caso español en el contexto internacional». En: Pazos, M. (dir.). Economía e igualdad de género: Retos de la Hacienda Pública en el siglo XXI. Madrid: Instituto de Estudios Fiscales. Colección Estudios de Hacienda Pública, 161-18.

ESPING-ANDERSEN, G. y SARASA, S. (2002). "The gendered conflict reconsidered». Journal of European Social Policy, 12, 5-21.

EuROSTAT (2009). The provision of childcare services: A comparative review of 30 European countries. European Communities.

FERNÁNDEZ, R. y FogLI, A. (2009). "Culture: An Empirical Investigation of Beliefs, Work and Fertility». American Economic Journal: Macroeconomics, 1 (1), 146-77.

Fernández, R.; Fogli, A. y Olivetti, C. (2004). «Preference Formation and the Rise of Women's Labour Force Participation, Evidence from WWII». CEPR Discussion Papers, 4493.

Feyrer, J.; Sacerdote, B. y Stern, A. (2008). "Will the stork return to Europe and Japan?: Understanding fertility within developed nations». The Journal of Economic Perspectives, 22 (3), 3-22.

FLAQUER, L. (2005). «Régimen de bienestar, familiarismo y ciudadanía». En: Solé, C. y FlaQUeR, L. (eds.). El uso de las políticas sociales por las mujeres inmigrantes. Madrid: Ministerio de Trabajo y Asuntos Sociales. Instituto de la Mujer, 21-52.

Flaquer, L.; Escobedo, A. y NaVArro, L. (2009). El impacto de la maternidad y la paternidad en el empleo: Itinerarios laborales de madres y padres en hogares con menores de tres años. Análisis de cambios y discontinuidades a partir de la muestra continua de vidas laborales. Madrid: FIPROS.

Gauthier, A. (2007). "The impact of family policies on fertility in industrialized countries, a review of the literature». Population Research and Policy Review, 26 (3), 323-346.

Goldscheider, F.; OlÁH, L. y PUUR, A. (2010). «Reconciling studies of men’s gender attitudes and fertility: Response to Westoff and Higgins Frances Goldscheider». Demographic Research [en línea], 22 (8), 189-198. <http,/www.demographicresearch.org/Volumes/Vol22/8/ DOI, 10.4054/DemRes.2010.22.8>.

GonZÁlEZ, M.J. y JuRADO, T. (2006). «Remaining childless in affluent economies: a comparison of France, West Germany, Italy and Spain, 1994-2001». European Journal of Population, 22 (4), 317-352.

- (2009). "¿Cuándo se implican los hombres en las tareas domésticas? Un análisis de la Encuesta de Empleo del Tiempo». Revista Panorama Social, 65-81.

GornicK, J.; MEYERS, M. y Ross, K. (1997). «Supporting the employment of mothers: Policy variation across fourteen welfare states». Journal of European Social Policy, 7 (1), 45-70.

GÜell, M.; Brodman, S. y Esping-Andersen, G. (2007). "When Fertility is Bargained, Second Births in Denmark and Spain». European Sociological Review, 23 (5), 599-613. 
Gustafsson, S. y KALWIJ, A. (eds.) (2006). Education and Postponement of Maternity. Economic Analyses for Industrialized Countries: European Studies of Population. Berlín: Springer.

GuTIÉRREZ-DOMÈNECH, M. (2008). «The impact of the labour market on the timing of marriage and births in Spain». Journal of Population Economics, 21 (1), 83-110.

Hakim, C. (2000). Work-lifestyle choices in the 21 st century: Preference theory. Oxford: Oxford University Press.

- (2005). Modelos de familia en las sociedades modernas. Madrid: Centro de Investigaciones Sociológicas.

Kohler, H.P.; Billari, F. y Ortega, J. (2006). «Low Fertility in Europe: Causes, Implications and Policy Options». En: Harris, F.R. (ed.). The Baby Bust: Who Will Do the Work? Who Will Pay the Taxes? United States: Rowman \& Littlefield Publishers, Lanham, 48-109.

LEITNER, S. (2003). «Varieties of familism, the caring function of the family in comparative perspective». European Societies, 5 (4), 353-375.

LEWIS, J. (1992). "Gender and the development of welfare regimes». Journal of European Social Policy, 2 (3), 159-173.

- (2002). «Individualisation, assumptions about the existence of an adult worker model and the shift towards contractualism». En: CARLING, A.; DunCAN, S. and EDWards, R. (eds.). Analysing Families: Morality and Rationality in Policy and Practice. London: Routledge, 51-56.

López Blasco, A. y GiL, G. (2008). Informe de la Juventud 2008. Tomo I. Jóvenes en una sociedad cambiante: Demografía y transiciones a la vida adulta. Madrid: Ministerio de Igualdad.

LÜCK, D. y HOFÄCKER, D. (2008). "The values of work and care among women in modern societies». En: Pfau-Effinger, Birgit; OpielKa, Michael y Van OOrSСНОт, Wim (Hrsg.). Culture and Welfare State: The Values of Social Policy. Cheltenham: Edward Elgar.

MARTÍN GARCÍA, T. (2009). "A reassessment of the role of women's education in existing fertility research». Genus, LXIV (1-2), 101-129.

- (2010). "The impact of occupational sex-composition on women's fertility in Spain». European Societies, 12 (1), 113-134.

MATYSIAK, A. Y VignOLI, D. (2006). Fertility and women's employment: a meta-analysis, MPIDR Working Paper WP-2006-048, Rostock: Max Planck Institute for Demographic Research

MCDONALD, P. (2000). "Gender equity, social institutions and the future of fertility». Journal of Population Research, 17, 1-16.

MeIL, G. (2006). "Welfare Policies, Work and Family Lives in Modern Spain». En: Rossi, G. (ed.). Reconciling Family and Work: New Challenges for Social Policies in Europe. Milán: Franco Angeli, 37-58.

Michaud, P. y Tatsiramos, K. (2005). «Employment and dynamics of married women in Europe». Paper of IZA, 1706. Institute for the Study Labor.

Moreno Mínguez, A. (2007). Familia y empleo de la mujer en los estados del bienestar del sur de Europa: Incidencia de las politicas familiares y laborales. Madrid: Centro de Investigaciones Sociológicas. Colección Monografías.

- (2008). «El reducido empleo femenino en los estados del bienestar del Sur de Europa, un análisis comparado». Revista Internacional de Sociología, 50, 126-162.

- (2010). "Family and gender roles in Spain from a comparative perspective». European Societies, 12 (1), 85-112. 
Naldini, M. (2003). The Family in the Mediterranean Welfare States. Londres / Portland: Frank Cass.

NeYer, G. (2003). «Family policies and low fertility in Western Europe». MPIDR Working Paper, WP 2003-021. Rostock: Max Planck Institute for Demographic Research.

OCDE (2007). Statistical Database [en línea]. <http,//stats.oecd.org/wbos/Default. aspx? usercontext=sourceoecd $>$ [Consulta: febrero 2008].

OlÁH, L. (2003). «Gendering fertility, second births in Sweden and Hungary». Population Research and Policy Review, 22, 171-200.

Parella, S. (2008). Informe de la Juventud 2008. Tomo 5. Desigualdades de género. Jóvenes inmigrantes. Madrid: Ministerio de Igualdad.

PASCALl, G. y LEWIS, J. (2001). "Care work beyond Beveridge Benefits». The Journal of Poverty and Social Justice, 32, 1-6.

Pfau-Effinger, B. (2005). "Culture and Welfare State Policies: Reflections on a Complex Interrelation». Journal of Social Policy, 34 (1), 3-20.

Puur, A.; Olah, L.S.; Tazi-Preve, M.I. y Dorbritz, J. (2008). «Men's childbearing desires and views of the male role in Europe at the dawn of the $21 \mathrm{st}$ century». Demographic Research, 19 (56), 1883-1912. doi:10.4054/DemRes.2008.19.56.

RanCI, C. (2010). Social Vulnearability in Europe. The New Configurations of Social Ris., New York: Palgrave Macmillan

Rissman, B. (2004). «Gender as a Social Structure. Theory Wrestling with Activism». Gender \& Society, 18, 429-450.

Ronsen, M. y SKREDE, K. (2010). "Can public policies sustain fertility in the Nordic countries?: Lessons from the past and questions for the future». Demographic Research [en línea], 22 (13), 321-346. <http,//www.demographic-research.org/ Volumes/Vol22/13/ DOI, 10.4054/DemRes.2010.22.13>.

Sainsbury, D. (ed.) (1999). Gender and Welfare State Regimes. Oxford: Oxford University Press.

SeVilla Sanz, A. y De LaAT, J. (2006). Working women, men's home time and lowestlow fertility. ISER Working Paper 2006-23. University of Essex. Institute for Social and Economic Research.

SOLERA, C. (2009). Women in and out of paid work: Changes across generations in Italy and Britain. Bristol: The Policy Press.

Thévenon, O. (2009). "Assessing the cost of children: A challenge for policies». En: Letablier, M.; Luci, A.; Math, A. y Thévenon, O. (eds.). The Costs of Raising Children and the Effectiveness of Supporting Parenthood Policies in European Countries: A Literature Review. Rapport pour la Commission Européenne, INED.

TORR, B.M. y SHORT, S.E. (2004). «Second births and the second shift, a research note on gender equity and fertility». Population and Development Review, 30, 109-130.

VALIENTE, C. (2010). "The erosion of "familism" in the Spanish Welfare State: childcare policy since 1975». En: AJZENSTADT, M. y GAL, J. (ed.). Children, gender and families in Mediterranean Welfare States. Londres: Springer.

VAN DE KAA, D. (1987), «Europe's Second Demographic Transition». Population Bulletin, 42 (1), 3-24.

ViCENÇ, N. (2006). El subdesarrollo social de España: Causas y consecuencias. Barcelona: Anagrama. 
WALL, K. (2007). Main patterns in attitudes to the articulation between work and family life: A cross-national analysis, in Families, States and Labour Markets. Institutions, causes and consequences of family policies in Post-War Welfare States. Glos: Edward Elgar.

West, C. y Zimmerman, D.H. (1987). «Doing gender». Gender \& Society, 1 (2), 125-151.

Westoff, C. y Higgins, J. (2009). «Relationships between men’s gender attitudes and fertility: Response to Puur et al.'s "Men's childbearing desires and views of the male role in Europe at the dawn of the 21st century"». Demographic Research, 21 (3), 65-74. doi:10.4054/DemRes.2009.21.3.

ZuannA, D. (2001). «The banquet of Aeolus: A familistic interpretation of Italy's lowest low fertility». Demographic Research [en línea], 4. <www.demographicresearch.org>.

ZuANNA, D. y Micheli, G. (eds.) (2004). «Strong family and low fertility, a paradox?: New perspectives in interpreting contemporary family and reproductive behaviour Series». European Studies of Population, 14.

\section{Agradecimientos}

Este artículo se ha realizado en el marco del proyecto I+D no 151/06 del Instituto de la Mujer y del proyecto FIPROS 2005-134 del Ministerio de Trabajo e Inmigración. Agradezco a las evaluadoras y a los evaluadores externos, así como a los editores, las valiosas aportaciones realizadas. 